\title{
TWISTED GROUPS AND LOCALLY TOROIDAL REGULAR POLYTOPES
}

\author{
PETER MCMULLEN AND EGON SCHULTE
}

\begin{abstract}
In recent years, much work has been done on the classification of abstract regular polytopes by their local and global topological type. Abstract regular polytopes are combinatorial structures which generalize the wellknown classical geometric regular polytopes and tessellations. In this context, the classical theory is concerned with those which are of globally or locally spherical type. In a sequence of papers, the authors have studied the corresponding classification of abstract regular polytopes which are globally or locally toroidal. Here, this investigation of locally toroidal regular polytopes is continued, with a particular emphasis on polytopes of ranks 5 and 6 . For large classes of such polytopes, their groups are explicitly identified using twisting operations on quotients of Coxeter groups. In particular, this leads to new classification results which complement those obtained elsewhere. The method is also applied to describe certain regular polytopes with small facets and vertex-figures.
\end{abstract}

\section{INTRODUCTION}

In recent years, the classical notion of a regular polytope has been generalized to abstract regular polytopes; these are combinatorial structures with a distinctive geometric or topological flavour, which resemble the classical regular polytopes (Danzer-Schulte [8], McMullen-Schulte [23]). For related notions in geometric or group theoretic contexts, see also McMullen [12], Grünbaum [10], Dress [9], Buekenhout [1] and Tits [29].

A central problem in the classical theory is the complete description of all regular polytopes and tessellations in spherical, euclidean or hyperbolic space. The solution to this problem is well-known; see Coxeter $[4,5]$. When posed within the theory of abstract polytopes, the classification problem must necessarily take a different form, because a priori an abstract polytope is not embedded into an ambient space. A suitable substitute is now the classification by global or local topological type. Not every abstract polytope admits a natural topology, but if it does, then it is subject to classification with respect to this topology.

The classical theory solves the spherical case. Using terminology to be introduced in Section 2, this can be summarized by saying that the only universal abstract regular polytopes which are locally spherical are the classical regular tessellations in spherical, euclidean or hyperbolic space. Among these, only those that

Received by the editors January 7, 1995 .

1991 Mathematics Subject Classification. Primary 51M20.

Key words and phrases. Polyhedra and polytopes; regular figures, division of space.

Supported by NSF grant DMS-9202071. 
are globally spherical (isomorphic to convex regular polytopes) are finite (Coxeter [5], Schulte [25], McMullen-Schulte [23]). The next simplest topological kind of finite regular polytope is a toroid, and so a main problem is the corresponding classification in the locally toroidal case (we shall clarify what this means in Section 2). The problem of classifying the locally toroidal regular polytopes (of rank 4) was raised by Grünbaum [10] in the 70's; see also Coxeter-Shephard [7] and Weiss [31].

In a sequence of papers, the present authors have extensively studied the globally toroidal regular polytopes (regular toroids) and the locally toroidal regular polytopes. For a recent survey on this subject, see [28]. For rank 3, the regular toroids are the well-known regular (reflexible) maps $\{4,4\}_{(s, t)},\{6,3\}_{(s, t)}$ and $\{3,6\}_{(s, t)}$ (with $t=0$ or $t=s$ ) on the 2-torus (Coxeter-Moser [6]). In [21], the regular toroids of rank $n+1$ were classified for all $n$; they correspond to the regular tessellations on the $n$-torus (see Section 3 ). Then an abstract polytope is called locally toroidal if its facets and vertex-figures are (globally) spherical or toroidal, with at least one kind toroidal. Locally toroidal regular polytopes can only exist in ranks 4,5 and 6 , because in higher ranks there are no suitable hyperbolic honeycombs which cover them.

At present, the situation is best understood in ranks 4 and 5. In rank 4, the classification involves the study of each of the Schläfli types $\{4,4, r\}$ with $r=3,4$, $\{6,3, p\}$ with $p=3,4,5,6$, and $\{3,6,3\}$, and their duals. The classification is complete for all types except $\{4,4,4\}$ and $\{3,6,3\}([18,19])$. For $\{4,4,4\}$ it is nearly complete, but for $\{3,6,3\}$ only partial results are known. In the spherical case, each Schläfli symbol gives just one polytope. In the toroidal case, however, this is no longer true, and the classification must necessarily be carried out among all polytopes with given isomorphism types of facet and vertex-figure. Since the regular $(n+1)$-toroids are parametrized by $n$-vectors (like $(s, t)$ for $\left.\{6,3\}_{(s, t)}\right)$, this leads to a discussion of different classes of polytopes, each parametrized by one or two such vectors.

For instance, for the class $\mathcal{C}=\left\langle\{6,3\}_{(s, t)},\{3,3\}\right\rangle$ of locally toroidal regular 4polytopes with facets of type $\{6,3\}(s, t)$ and tetrahedral vertex-figures $\{3,3\}$, the classification reads as follows. For each $(s, t)$ with $s \geq 2$ and $t=0$ or $s$, there is a universal regular polytope in $\mathcal{C}$, denoted and uniquely determined by the generalized Schläfli symbol $\left\{\{6,3\}_{(s, t)},\{3,3\}\right\}$; this polytope is finite if and only if $(s, t)=$ $(2,0),(3,0),(4,0)$ or $(2,2)$. In particular, in the finite cases, the automorphism groups are $S_{5} \times C_{2}$ of order 240, [1 112$]^{3} \ltimes C_{2}$ of order 1296, [1 112$]^{4} \ltimes C_{2}$ of order

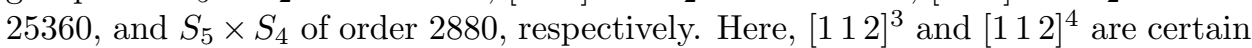
finite unitary reflexion groups in complex 4 -space $([3,18])$. There are similar such classification results for all of the above Schläfli types.

In rank 5, only the Schläfli type $\{3,4,3,4\}$ and its dual occur. In [21] it was shown that just three parameter vectors belong to finite universal polytopes. However, the structure of the corresponding group was not determined for two of these three. The two missing groups have now been found by an application of our approach in Section 7, Corollary 7.10 and Theorem 7.13; see also Corollary 7.4 for the third group. In rank 6 , the types are $\{3,3,3,4,3\},\{3,3,4,3,3\}$ and $\{3,4,3,3,4\}$, and their duals. The known finite polytopes are listed in [21]; these lists are conjectured to be complete, and this is supported by geometric arguments.

In this paper we again investigate the locally toroidal regular polytopes, now with a particular emphasis on the polytopes of ranks 5 and 6 . After a brief summary 
of the general properties of regular polytopes in Section 2, and a review of the toroids in Section 3, we discuss in Section 4 a stronger version of some twisting arguments which occurred in [16]. We construct certain regular polytopes $2^{\mathcal{K}, \mathcal{G}}$ and $\mathcal{L}^{\mathcal{K}, \mathcal{G}}$, whose groups are semi-direct products of the Coxeter group with diagram $\mathcal{G}$ by the group of their vertex-figure or co-faces $\mathcal{K}$, respectively. It is striking that many universal locally toroidal regular polytopes are indeed such polytopes $2^{\mathcal{K}, \mathcal{G}}$ or $\mathcal{L}^{\mathcal{K}, \mathcal{G}}$ for suitable $\mathcal{L}, \mathcal{K}$ and $\mathcal{G}$. This fact rests on a corresponding universality property of these polytopes proved in Section 5, and in particular leads to some new classification results.

In Section 6 we present a new construction of locally toroidal regular 5-polytopes. In particular, this proves the non-finiteness of all universal 5-polytopes $\{\{3,4,3\}$, $\left.\{4,3,4\}_{\mathbf{s}}\right\}$ with "odd" parameter vectors $\mathbf{s}$. It also implies that each of these polytopes has infinitely many finite quotients with the same facets $\{3,4,3\}$ and vertexfigures $\{4,3,4\}_{\mathbf{s}}$. Finally, in Section 7 we describe a method of finding polytopes with small faces as quotients of polytopes constructed in earlier sections. This also includes the recognition of the groups of regular polytopes of rank 6 whose facets and vertex-figures are small toroids of rank 5; see Corollary 7.11 for an example. The method is strong enough to work for some other topological types as well. For example, Theorem 7.14 deals with some polytopes of "mixed toroidal-projective type".

\section{BASIC NOTIONS}

In this section we give a brief introduction to the theory of abstract regular polytopes. For more details the reader is referred to $[15,23]$.

An (abstract) polytope of rank $n$, or simply an $n$-polytope, is a partially ordered set $\mathcal{P}$ with a strictly monotone rank function with range $\{-1,0, \ldots, n\}$. The elements of rank $i$ are called the $i$-faces of $\mathcal{P}$, or vertices, edges and facets of $\mathcal{P}$ if $i=0,1$ or $n-1$, respectively. The flags (maximal totally ordered subsets) of $\mathcal{P}$ all contain exactly $n+2$ faces, including the unique minimal face $F_{-1}$ and unique maximal face $F_{n}$ of $\mathcal{P}$. Further, $\mathcal{P}$ is strongly flag-connected, meaning that any two flags $\Phi$ and $\Psi$ of $\mathcal{P}$ can be joined by a sequence of flags $\Phi=\Phi_{0}, \Phi_{1}, \ldots, \Phi_{k}=\Psi$, which are such that $\Phi_{i-1}$ and $\Phi_{i}$ are adjacent (differ by one face), and such that $\Phi \cap \Psi \subseteq \Phi_{i}$ for each $i$. Finally, if $F$ and $G$ are an $(i-1)$-face and an $(i+1)$-face with $F<G$, then there are exactly two $i$-faces $H$ such that $F<H<G$.

When $F$ and $G$ are two faces of a polytope $\mathcal{P}$ with $F \leq G$, we call $G / F:=$ $\{H \mid F \leq H \leq G\}$ a section of $\mathcal{P}$. We may usually safely identify a face $F$ with the section $F / F_{-1}$. For a face $F$ the section $F_{n} / F$ is called the co-face of $\mathcal{P}$ at $F$, or the vertex-figure at $F$ if $F$ is a vertex.

An abstract $n$-polytope $\mathcal{P}$ is regular if its (combinatorial automorphism) group $A(\mathcal{P})$ is transitive on its flags. Let $\Phi:=\left\{F_{-1}, F_{0}, \ldots, F_{n-1}, F_{n}\right\}$ be a fixed or base flag of $\mathcal{P}$; occasionally we do not mention $F_{-1}$ and $F_{n}$, because they belong to each flag. The group $A(\mathcal{P})$ of a regular $n$-polytope $\mathcal{P}$ is generated by distinguished generators $\rho_{0}, \ldots, \rho_{n-1}$ (with respect to $\left.\Phi\right)$, where $\rho_{i}$ is the unique automorphism which keeps all but the $i$-face of $\Phi$ fixed. These generators satisfy relations

$$
\left(\rho_{i} \rho_{j}\right)^{p_{i j}}=\varepsilon \quad(i, j=0, \ldots, n-1),
$$

$$
p_{i i}=1, \quad p_{i j}=p_{j i} \geq 2 \quad(i \neq j)
$$


and

$$
p_{i j}=2 \text { if }|i-j| \geq 2 .
$$

A coarse description of the combinatorial properties of $\mathcal{P}$ is given by its (Schläfli) type $\left\{p_{1}, \ldots, p_{n-1}\right\}$, whose entries are the numbers $p_{j}:=p_{j-1, j}(j=1, \ldots$, $n-1$ ). Further, $A(\mathcal{P})$ has the intersection property (with respect to the distinguished generators), namely

$$
\left\langle\rho_{i} \mid i \in I\right\rangle \cap\left\langle\rho_{i} \mid i \in J\right\rangle=\left\langle\rho_{i} \mid i \in I \cap J\right\rangle \text { for all } I, J \subset\{0, \ldots, n-1\} .
$$

By a $C$-group we mean a group which is generated by involutions such that (1), (2) and (4) hold. If in addition (3) holds, then the group is called a string C-group. The automorphism group of a regular polytope is a string C-group. Conversely, given a string C-group, there is an associated regular polytope of which it is the automorphism group $([25,15])$. Note that Coxeter groups are examples of C-groups ([29]).

We remark that our usage of the term "C-group" differs from that in previous papers, where the term was used for groups which we now call string C-groups. From now on we include the qualification "string" to emphasize that a string Cgroup is a quotient of the Coxeter group $\left[p_{1}, \ldots, p_{n-1}\right]$ with the string diagram

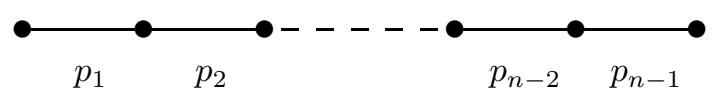

with the $p_{j}$ defined as above. Note that $\left[p_{1}, \ldots, p_{n-1}\right]$ is the group of the universal regular polytope $\left\{p_{1}, \ldots, p_{n-1}\right\}$ ([25]). For example, $[6,3,3]$ is the group of the regular honeycomb $\{6,3,3\}$ in hyperbolic 3 -space $([4])$.

Let $\mathcal{P}$ and $\mathcal{Q}$ be $n$-polytopes. A mapping $\varphi: \mathcal{P} \rightarrow \mathcal{Q}$ is called a covering if $\varphi$ is incidence- and rank-preserving, and maps adjacent flags onto (distinct) adjacent flags; then $\varphi$ is necessarily surjective. A covering $\varphi$ is called a $k$-covering if it maps sections of $\mathcal{P}$ of rank at most $k$ by an isomorphism onto corresponding sections of $\mathcal{Q}$. If $\varphi: \mathcal{P} \rightarrow \mathcal{Q}$ is a covering, then we say that $\mathcal{P}$ covers $\mathcal{Q}$, or that $\mathcal{Q}$ is covered by $\mathcal{P}$.

Let $\mathcal{P}$ be an $n$-polytope and $N$ a subgroup of $A(\mathcal{P})$. We denote by $\mathcal{P} / N$ the set of orbits of $N$ in $\mathcal{P}$; the orbit of a face $F$ of $\mathcal{P}$ is written $N \cdot F$. We introduce a partial order on $\mathcal{P} / N$ as follows: if $G_{1}, G_{2} \in \mathcal{P} / N$, then $G_{1} \leq G_{2}$ if and only if $G_{i}=N \cdot F_{i}$ for some $F_{i} \in \mathcal{P}(i=1,2)$ with $F_{1} \leq F_{2}$. The set $\mathcal{P} / N$ together with this partial order is called the quotient of $\mathcal{P}$ with respect to $N$. In general, $\mathcal{P} / N$ will not be a polytope, but if it is, then the canonical projection $\pi_{N}: F \mapsto$ $N \cdot F$ defines a covering of $\mathcal{P} / N$ by $\mathcal{P}$. In particular, if $\mathcal{P}$ is regular and $N$ a normal subgroup of $A(\mathcal{P})=\left\langle\rho_{0}, \ldots, \rho_{n-1}\right\rangle$ such that $A(\mathcal{P}) / N$ is a string C-group (with generators $\left.\rho_{0} N, \ldots, \rho_{n-1} N\right)$, then $\mathcal{P} / N$ is a regular $n$-polytope with group $A(\mathcal{P} / N) \simeq A(\mathcal{P}) / N$ (under an isomorphism mapping distinguished generators to distinguished generators). For further details, see [22, 23].

In verifying that a given group is a C-group, it is usually only the intersection property which causes difficulty. Here the following result is sometimes useful; we shall refer to it as the quotient lemma ([22]).

Lemma 2.1. Let $U:=\left\langle\rho_{0}, \ldots, \rho_{n-1}\right\rangle$ be a group generated by involutions $\rho_{0}, \ldots$, $\rho_{n-1}$, such that $\left(\rho_{i} \rho_{j}\right)^{2}=\varepsilon$ whenever $j-i \geq 2$, and let $\left\langle\sigma_{0}, \ldots, \sigma_{n-1}\right\rangle$ be a string 
$C$-group with respect to the distinguished generators $\sigma_{0}, \ldots, \sigma_{n-1}$. If the mapping $\rho_{j} \mapsto \sigma_{j}$ for $j=0, \ldots, n-1$ defines a homomorphism which is one-to-one on $\left\langle\rho_{0}, \ldots, \rho_{n-2}\right\rangle$ or on $\left\langle\rho_{1}, \ldots, \rho_{n-1}\right\rangle$, then $U$ is also a string $C$-group.

In other words, $U$ is the group of a regular $n$-polytope, and this polytope covers the regular $n$-polytope with group $\left\langle\sigma_{0}, \ldots, \sigma_{n-1}\right\rangle$.

The quotient lemma is an important tool in the investigation of one of the main problems in the theory, that of the amalgamation of regular polytopes of lower rank. Given regular $n$-polytopes $\mathcal{P}_{1}$ and $\mathcal{P}_{2}$ such that the vertex-figures of $\mathcal{P}_{1}$ are isomorphic to the facets of $\mathcal{P}_{2}$, we denote by $\left\langle\mathcal{P}_{1}, \mathcal{P}_{2}\right\rangle$ the class of all regular $(n+1)$ polytopes $\mathcal{P}$ with facets isomorphic to $\mathcal{P}_{1}$ and vertex-figures isomorphic to $\mathcal{P}_{2}$. If $\left\langle\mathcal{P}_{1}, \mathcal{P}_{2}\right\rangle \neq \emptyset$, then any such $\mathcal{P}$ is a quotient of a universal member of $\left\langle\mathcal{P}_{1}, \mathcal{P}_{2}\right\rangle$, the universal $(n-1)$-cover of $\mathcal{P}$; this universal polytope is denoted by $\left\{\mathcal{P}_{1}, \mathcal{P}_{2}\right\}([26])$.

For instance, if $\mathcal{P}_{1}$ is the octahedron $\{3,4\}$ and $\mathcal{P}_{2}$ the cube $\{4,3\}$, then $\left\langle\mathcal{P}_{1}, \mathcal{P}_{2}\right\rangle$ consists of all abstract regular 4-polytopes with octahedral facets and cubical vertexfigures. The universal polytope $\left\{\mathcal{P}_{1}, \mathcal{P}_{2}\right\}$ is now the (finite) regular 24 -cell $\{3,4,3\}$ with group $[3,4,3]$ of order 1152 . This covers all the polytopes in $\left\langle\mathcal{P}_{1}, \mathcal{P}_{2}\right\rangle$, whose only other member is the polytope $\{3,4,3\}_{6}$ (which we shall meet again below), obtained by identifying antipodal faces of the 24-cell of each dimension (rank). On the other hand, if $\mathcal{P}_{1}=\{4,3\}$ and $\mathcal{P}_{2}=\{3,4\}$, then $\left\{\mathcal{P}_{1}, \mathcal{P}_{2}\right\}$ is the (infinite) tessellation $\{4,3,4\}$ of euclidean 3 -space by cubes; the toroids $\{4,3,4\}_{\mathbf{s}}$ defined in the next section are then non-universal members in the class $\left\langle\mathcal{P}_{1}, \mathcal{P}_{2}\right\rangle$.

These two examples of spherical and locally spherical polytopes illustrate the following problems about general universal polytopes. When is $\left\langle\mathcal{P}_{1}, \mathcal{P}_{2}\right\rangle$ non-empty, or, equivalently, when does $\left\{\mathcal{P}_{1}, \mathcal{P}_{2}\right\}$ exist? When is $\left\{\mathcal{P}_{1}, \mathcal{P}_{2}\right\}$ finite? How can we construct $\left\{\mathcal{P}_{1}, \mathcal{P}_{2}\right\}$ and its group? In this paper, what we have in mind when we use the term "classification" of polytopes is the classification of all finite universal polytopes in the given context. It would of course be desirable actually to describe all the polytopes in a class $\left\langle\mathcal{P}_{1}, \mathcal{P}_{2}\right\rangle$. However, in view of the results of [20], this seems to be rather hopeless. In fact, very often the class contains infinitely many finite polytopes if it contains a suitable infinite polytope. We shall elaborate on this in Section 6.

An abstract regular $(n+1)$-polytope in $\left\langle\mathcal{P}_{1}, \mathcal{P}_{2}\right\rangle$ is called locally spherical if $\mathcal{P}_{1}$ and $\mathcal{P}_{2}$ are isomorphic to (classical) regular convex polytopes. It is locally toroidal if $\mathcal{P}_{1}$ and $\mathcal{P}_{2}$ are isomorphic to regular convex polytopes or regular toroids, with at least one of the latter kind. If $\mathcal{P}_{1}$ and $\mathcal{P}_{2}$ are of Schläfli types $\left\{p_{1}, p_{2}, \ldots, p_{n-1}\right\}$ and $\left\{p_{2}, \ldots, p_{n-1}, p_{n}\right\}$ respectively, then any locally toroidal $(n+1)$-polytope in $\left\langle\mathcal{P}_{1}, \mathcal{P}_{2}\right\rangle$ is a quotient of the regular honeycomb $\left\{p_{1}, \ldots, p_{n}\right\}$, which is in hyperbolic $n$-space. Since there are no such honeycombs in dimensions greater than 5 , this limits the classification of the corresponding polytopes to ranks 4, 5 and 6 (see [4]).

\section{REgUlar TOROIDS}

In this section, we briefly summarize results on globally toroidal regular polytopes or, briefly, the regular toroids. The regular toroids of rank $n+1$ are obtained as quotients of a regular honeycomb in euclidean $n$-space $E^{n}$ by normal subgroups or identification lattices of their translational symmetries. The regular toroids of rank 3 are well-known, and correspond to the regular maps on the 2-torus (CoxeterMoser [6]). These are the maps $\{4,4\}_{(s, t)},\{6,3\}_{(s, t)}$ and $\{3,6\}_{(s, t)}$ with $t=0$ or $t=s$. 
The classification problem for higher ranks was solved in [21]. Apart from rank $n=5$, the only examples come from the cubical honeycomb $\left\{4,3^{n-2}, 4\right\}$ in $E^{n}$ for $n \geq 2$, which gives rise to the regular $(n+1)$-toroids $\left\{4,3^{n-2}, 4\right\}_{\mathbf{s}}$, with $\mathbf{s}:=$ $\left(s^{k}, 0^{n-k}\right), s \geq 2$ and $k=1,2$ or $n$. In such contexts, the notation $q^{m}$ stands for a string $q, \ldots, q$ of length $m$. If $n=2$, these are the maps $\{4,4\}_{(s, 0)}$ and $\{4,4\}_{(s, s)}$ on the 2-torus, as above. In general, if we take the vertex set of the honeycomb to be the integer lattice $Z^{n}$, then the identification lattice $\Lambda_{\mathbf{s}}$ for $\left\{4,3^{n-2}, 4\right\}_{\mathbf{s}}$ is spanned by $\mathbf{s}$ and all vectors obtained from $\mathbf{s}$ by permuting its coordinates and changing their signs.

For $n \geq 3$ there are exactly three classes of toroids parametrized by $k$; for $n=2$ two of these classes coincide. The group of the regular toroid $\left\{4,3^{n-2}, 4\right\}_{\mathbf{s}}$ is denoted by $\left[4,3^{n-2}, 4\right]_{\mathbf{s}}$, which is the Coxeter group $\left[4,3^{n-2}, 4\right]=\left\langle\rho_{0}, \ldots, \rho_{n}\right\rangle$, factored out by the single extra relation

$$
\left(\rho_{0} \rho_{1} \cdots \rho_{n} \rho_{n-1} \cdots \rho_{k}\right)^{s k}=\varepsilon
$$

where $\varepsilon$ throughout denotes the identity. The details are given in Table 1 ; in this and similar tables, $v$ and $f$ are the numbers of vertices and facets, respectively, while $g$ is the group order.

The only other regular toroids of rank 4 or more are dual pairs of regular 5toroids derived from the honeycombs $\{3,3,4,3\}$ and $\{3,4,3,3\}$. We just consider the former, and take the vertex-set of the honeycomb to be $Z^{4} \cup\left(Z^{4}+\left(\frac{1}{2}, \frac{1}{2}, \frac{1}{2}, \frac{1}{2}\right)\right)$, the set of points in $E^{4}$ whose cartesian coordinates are all integers or all halves of odd integers (or, equivalently, the set of points corresponding to the integral quaternions). Again, the identification lattice $\Lambda_{\mathbf{s}}$ is spanned by a vector $\mathbf{s}:=$ $\left(s^{k}, 0^{n-k}\right)$ and its transforms under the group of the vertex-figure at $(0, \ldots, 0)$, but this time the additional symmetries (induced by right and left multiplication by unit quaternions, and by conjugation of quaternions) imply that the case $k=4$ is already accounted for by the case $k=1$; more exactly, $(s, s, s, s)$ is equivalent to $(2 s, 0,0,0)$. This leaves only the two choices $k=1,2$, which indeed give regular toroids $\{3,3,4,3\}_{\mathbf{s}}$. The group of $\{3,3,4,3\}_{\mathbf{s}}$ is denoted by $[3,3,4,3]_{\mathbf{s}}$, which is the Coxeter group $[3,3,4,3]=\left\langle\rho_{0}, \ldots, \rho_{4}\right\rangle$, factored out by the relation

$$
\left\{\begin{array}{ccc}
\left(\rho_{0} \sigma \tau \sigma\right)^{s} & =\varepsilon \text { if } k=1 \\
\left(\rho_{0} \sigma \tau\right)^{2 s} & =\varepsilon \text { if } k=2,
\end{array}\right.
$$

where $\sigma:=\rho_{1} \rho_{2} \rho_{3} \rho_{2} \rho_{1}$ and $\tau:=\rho_{4} \rho_{3} \rho_{2} \rho_{3} \rho_{4}$. The details are given in Table 2; note that the group order in case $\mathbf{s}=\left(s^{k}, 0^{4-k}\right)$ can more succinctly be written as $g=1152 k^{2} s^{4}$, with analogous common expressions for $v$ and $f$.

TABLE 1 . The polytopes $\left\{4,3^{n-2}, 4\right\}_{\mathbf{s}}$.

\begin{tabular}{||c|c|c|c||}
\hline $\mathbf{S}$ & $v$ & $f$ & $g$ \\
\hline \hline$\left(s, 0^{n-1}\right)$ & $s^{n}$ & $s^{n}$ & $(2 s)^{n} \cdot n !$ \\
$\left(s^{2}, 0^{n-2}\right)$ & $2 s^{n}$ & $2 s^{n}$ & $2^{n+1} s^{n} \cdot n !$ \\
$\left(s^{n}\right)$ & $2^{n-1} s^{n}$ & $2^{n-1} s^{n}$ & $2^{2 n-1} s^{n} \cdot n !$ \\
\hline
\end{tabular}


TABLE 2. The polytopes $\{3,3,4,3\}_{\mathbf{s}}$.

\begin{tabular}{||c|c|c|c||}
\hline $\mathbf{s}$ & $v$ & $f$ & $g$ \\
\hline \hline$(s, 0,0,0)$ & $s^{4}$ & $3 s^{4}$ & $1152 s^{4}$ \\
$(s, s, 0,0)$ & $4 s^{4}$ & $12 s^{4}$ & $4608 s^{4}$ \\
\hline
\end{tabular}

\section{TWisting}

In [16], we described various methods of twisting Coxeter groups or unitary groups generated by involutory reflexions, and we applied these twisting techniques to the problem of classifying locally toroidal regular 4-polytopes. Since then, we have found that some of the assumptions we made were unnecessarily strong. We therefore now sketch an extension of the twisting technique (though not in its full generality); we shall apply it in later sections to determine the structure of the groups of certain regular polytopes.

The basic idea of twisting involves extending an existing C-group by taking its semi-direct product with a subgroup of its automorphism group, which will also be a C-group. In the present cases, the original group is given by a (generalized Coxeter) diagram, and the automorphisms will then be diagram automorphisms, which will usually act effectively only on some subset of the diagram.

We shall particularly concentrate on the construction of the regular polytopes $\mathcal{L}^{\mathcal{K}, \mathcal{G}}$, which are crucial for the next sections. Here, $\mathcal{L}$ and $\mathcal{K}$ are certain regular polytopes, and $\mathcal{G}$ is a Coxeter diagram on which $A(\mathcal{K})$ acts in a suitable way as a group of diagram symmetries. We shall frequently take $\mathcal{L}$ to be 1-dimensional; in this case, we also write $2^{\mathcal{K}, \mathcal{G}}$ for $\mathcal{L}^{\mathcal{K}, \mathcal{G}}$, since $\mathcal{L}$ is determined by its 2 vertices.

In our applications, a Coxeter diagram $\mathcal{G}$ will thus actually represent the corresponding Coxeter group. However, occasionally it is also useful to think of the diagram as representing a suitable quotient of the corresponding Coxeter group; indeed, under certain conditions, our construction of $\mathcal{L}^{\mathcal{K}, \mathcal{G}}$ carries over to this situation. In describing or drawing (Coxeter) diagrams $\mathcal{G}$ of groups generated by involutory reflexions, we adopt the following convention. It is often convenient to think of a pair of distinct nodes $i, j$ of $\mathcal{G}$ which are not directly joined in $\mathcal{G}$, and so correspond to commuting generators, as being joined by an improper branch with label $m_{i j}=2$. Accordingly, if a diagram $\mathcal{G}$ is drawn and some of its branches labelled 2, then these branches are improper and, strictly speaking, do not belong to $\mathcal{G}$.

The choice of diagrams will now be further restricted. Let $\mathcal{G}$ be a diagram of a group generated by involutory reflexions, and let $\mathcal{K}$ be a regular $n$-polytope with group $A(\mathcal{K})=\left\langle\tau_{0}, \ldots, \tau_{n-1}\right\rangle$. Then $\mathcal{G}$ is called $\mathcal{K}$-admissible if $A(\mathcal{K})$ acts as a group of diagram automorphisms on $\mathcal{G}$ with the following properties. First, $A(\mathcal{K})$ acts transitively on the set $V(\mathcal{G})$ of nodes of $\mathcal{G}$. Second, the subgroup $\left\langle\tau_{1}, \ldots, \tau_{n-1}\right\rangle$ of $A(\mathcal{K})$ stabilizes a node $F_{0}$ (say) of $\mathcal{G}$, the initial node (it may stabilize more than one such node). Third, with respect to $F_{0}$, the action of $A(\mathcal{K})$ respects the intersection property for the generators $\tau_{0}, \ldots, \tau_{n-1}$ in that, if $V(\mathcal{G}, I)$ denotes the set of transforms of $F_{0}$ under $\left\langle\tau_{i} \mid i \in I\right\rangle$ for $I \subseteq\{0, \ldots, n-1\}$, then

$$
V(\mathcal{G}, I) \cap V(\mathcal{G}, J)=V(\mathcal{G}, I \cap J)
$$

for all such $I$ and $J$. (In contrast to [16], we do not demand that $A(\mathcal{K})$ be faithfully represented as this group of diagram automorphisms.) Observe that, if $j \leq n-1$ 
and $\mathcal{K}_{j}$ is the basic $j$-face of $\mathcal{K}$, then the induced subdiagram of $\mathcal{G}$ with node set $V(\mathcal{G},\{0, \ldots, j-1\})$ is a $\mathcal{K}_{j}$-admissible diagram (with the same initial node).

We shall usually have $V(\mathcal{G})=V(\mathcal{K})$, the vertex set of $\mathcal{K}$; then $A(\mathcal{K})$ acts in the natural way, and $F_{0}$ is the vertex in the base flag of $\mathcal{K}$. In this situation, (7) is always satisfied, and $\mathcal{K}$-admissibility of a diagram $\mathcal{G}$ just means that $A(\mathcal{K})$ acts on $\mathcal{G}$ as a group of diagram automorphisms.

For instance, consider the diagram $\mathcal{G}$ on the vertex set of the octahedron $\mathcal{K}=$ $\{3,4\}$ which connects antipodal vertices by a branch labelled $s$ with $s \geq 3$, but has no other proper branches (or, equivalently, all other branches are improper). Then $A(\mathcal{K})$ acts on $\mathcal{G}$ in a natural way as a group of diagram symmetries; equivalently, $A(\mathcal{K})$ acts on the corresponding Coxeter group $D_{s} \times D_{s} \times D_{s}$ as a group of automorphisms permuting the generators. If the vertices of $\mathcal{K}$ are $1, \ldots, 6$ with $i, i+3(\bmod 6)$ antipodal, then we can take $\tau_{0}:=\left(\begin{array}{lll}1 & 2\end{array}\right)\left(\begin{array}{ll}4 & 5\end{array}\right), \tau_{1}:=\left(\begin{array}{lll}2 & 3\end{array}\right)\left(\begin{array}{ll}5 & 6\end{array}\right)$, $\tau_{2}:=(36)$, and $F_{0}:=1$. Then $F_{0}$ is fixed by the group $\left\langle\tau_{1}, \tau_{2}\right\rangle$ of the vertex-figure at $F_{0}$, and (7) is also satisfied. Hence $\mathcal{G}$ is $\mathcal{K}$-admissible. If we allow $s=2$, then $\mathcal{G}$ is the trivial diagram with no (proper) branches. We shall revisit this example in Corollary 5.8 below.

We construct the regular polytope $\mathcal{L}^{\mathcal{K}, \mathcal{G}}$ as follows. Let $\mathcal{K}$ and $\mathcal{G}$ be as above, and let $\mathcal{L}=\left\{q_{1}, \ldots, q_{m-1}\right\}$, the universal regular $m$-polytope whose group is $\left[q_{1}, \ldots, q_{m-1}\right]=\left\langle\sigma_{0}, \ldots, \sigma_{m-1}\right\rangle$ with string diagram

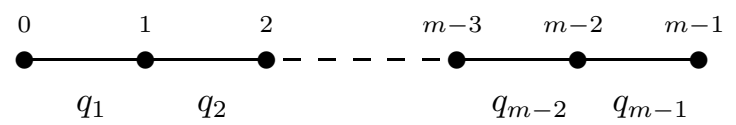

We shall construct a diagram $\mathcal{D}$ by adjoining $(8)$ to $\mathcal{G}$ as follows. We identify the node $m-1$ of $(8)$ with the node $F_{0}$ in $V(\mathcal{G})$, and take as the node set of $\mathcal{D}$ the disjoint union $V(\mathcal{D}):=V(\mathcal{G}) \cup\{0, \ldots, m-2\}$. As branches and labels of $\mathcal{D}$ we take all the old branches and labels of both $(8)$ and $\mathcal{G}$, and, in addition, for each node $F \neq F_{0}(=m-1)$ in $V(\mathcal{G})$, one new branch with label $q_{m-1}$ connecting $F$ to the node $m-2$ of $\mathcal{D}$. Then $\mathcal{D}$ is the diagram of a Coxeter group $W:=W(\mathcal{D})=$ $\left\langle\sigma_{k} \mid k \in V(\mathcal{D})\right\rangle$ on which $A(\mathcal{K})$ acts as a group of automorphisms permuting the generators. Note that $\mathcal{D}=\mathcal{G}$ (with initial node $F_{0}=0$ ) if $\mathcal{L}$ is of rank 1 .

In the above example with $\mathcal{K}=\{3,4\}$, if $\mathcal{L}$ is the triangle $\{3\}$, then $\mathcal{D}$ consists of three triangles with a common vertex $F_{0}=1$, and the branches of $\mathcal{D}$ are marked 3 or $s$ depending on whether or not the branch contains $F_{0}$.

We can now define the $(m+n)$-polytope $\mathcal{L}^{\mathcal{K}, \mathcal{G}}$ by its group $W \ltimes A(\mathcal{K})$ (the semi-direct product induced by the action of $A(\mathcal{K})$ on $W)$, and the distinguished generators $\rho_{0}, \ldots, \rho_{m+n-1}$ given by

$$
\rho_{j}:= \begin{cases}\sigma_{j} & \text { for } j=0, \ldots, m-1 \\ \tau_{j-m} & \text { for } j=m, \ldots, m+n-1 .\end{cases}
$$

Again, as in [16, p.215], this defines a string C-group. If $\mathcal{K}$ is of type $\left\{p_{1}, \ldots, p_{n-1}\right\}$, then $\mathcal{L}^{\mathcal{K}, \mathcal{G}}$ is of type $\left\{q_{1}, \ldots, q_{m-1}, 2 r, p_{1}, \ldots, p_{n-1}\right\}$, where $r$ is the mark of the (possibly improper) branch of $\mathcal{G}$ connecting the two nodes in $V(\mathcal{G},\{0\})=$ $\left\{F_{0}, \tau_{0}\left(F_{0}\right)\right\}$, the vertices of the base edge of $\mathcal{K}$. The $m$-faces of $\mathcal{L}^{\mathcal{K}, \mathcal{G}}$ are isomorphic to $\mathcal{L}$, and the co-faces at $(m-1)$-faces are isomorphic to $\mathcal{K}$. For $1 \leq j \leq n$, the $(m+j)$-faces of $\mathcal{L}^{\mathcal{K}, \mathcal{G}}$ are isomorphic to $\mathcal{L}^{\mathcal{K}_{j}, \mathcal{G}_{j}}$, where $\mathcal{K}_{j}$ is the basic $j$-face 
of $\mathcal{K}$ and $\mathcal{G}_{j}$ is the induced subdiagram of $\mathcal{G}$ on $V(\mathcal{G},\{0, \ldots, j-1\})$. Similarly, for $-1 \leq j \leq m-2$ the co-faces at $j$-faces of $\mathcal{L}^{\mathcal{K}, \mathcal{G}}$ are isomorphic to $\mathcal{L}_{j}^{\mathcal{K}, \mathcal{G}}$, with $\mathcal{L}_{j}=\left\{q_{j+2}, \ldots, q_{m-1}\right\}$.

Note that, in the above, we do not rule out the possibility that $\mathcal{K}$ is flat, where we recall that a polytope is called flat if each of its vertices is incident with each facet. In this case, if $V(\mathcal{G})=V(\mathcal{K})$ (with the natural action of $A(\mathcal{K})$ ), then $\mathcal{G}_{n-1}=\mathcal{G}$; moreover, $\mathcal{L}^{\mathcal{K}, \mathcal{G}}$ is also flat, and the facets of $\mathcal{L}^{\mathcal{K}, \mathcal{G}}$ are in one-to-one correspondence with the facets of $\mathcal{K}$.

Similarly, we do not exclude the case that $\mathcal{K}$ is neighbourly, where we recall that a polytope is neighbourly if any two of its vertices are joined by an edge. (More strictly, this property is 2-neighbourliness, but we shall not need to consider its higher generalizations here.) If $\mathcal{K}$ is neighbourly and regular, then any two vertices are joined by the same number of edges. But note that in a diagram $\mathcal{G}$ with $V(\mathcal{G})=V(\mathcal{K})$ only one of these edges is represented by a branch.

In most applications, $\mathcal{L}$ will be 1 -dimensional. In this case we write $2^{\mathcal{K}, \mathcal{G}}$ for $\mathcal{L}^{\mathcal{K}, \mathcal{G}}$. Further, if $\mathcal{G}$ is the trivial diagram on the vertex set $V(\mathcal{K})$ of $\mathcal{K}$, we simply write $\mathcal{L}^{\mathcal{K}}$ or $2^{\mathcal{K}}$ instead of $\mathcal{L}^{\mathcal{K}, \mathcal{G}}$ or $2^{\mathcal{K}, \mathcal{G}}$.

The following examples illustrate these concepts. In all cases, $\mathcal{G}$ will be the trivial diagram on $\mathcal{K}$, so that $\mathcal{L}^{\mathcal{K}, \mathcal{G}}=\mathcal{L}^{\mathcal{K}}$. In particular, if $\mathcal{L}$ is the triangle $\{3\}$ and $\mathcal{K}$ is the regular 4 -simplex $\{3,3,3\}$ (so that $\mathcal{G}$ has 5 vertices), we have

$$
\begin{aligned}
& \{3,4,3,3,3\}=\{3\}^{\{3,3,3\}}, \\
& {[3,4,3,3,3]=W(\mathcal{D}) \ltimes S_{5},}
\end{aligned}
$$

with $\mathcal{D}$ the Coxeter diagram

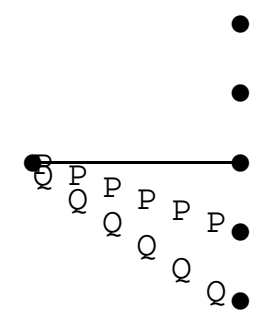

Similarly, if $\mathcal{L}$ and $\mathcal{K}$ are both the tetrahedron $\{3,3\}$ (with 4 vertices), we obtain

$$
\begin{aligned}
& \{3,3,4,3,3\}=\{3,3\}^{\{3,3\}}, \\
& {[3,3,4,3,3]=W(\mathcal{D}) \ltimes S_{4},}
\end{aligned}
$$

and now the same diagram $\mathcal{D}$ occurs in the form

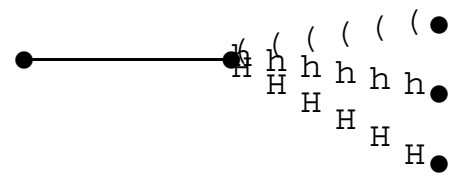

Finally, taking this diagram $\mathcal{D}$ with $W(\mathcal{D})=\left\langle\sigma_{0}, \ldots, \sigma_{5}\right\rangle$ in the form 


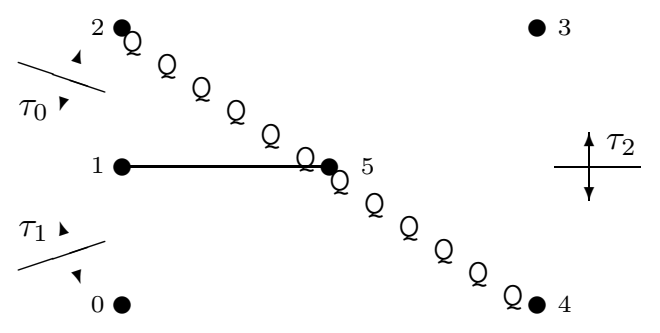

then the twisting operation

$$
\left(\sigma_{0}, \ldots, \sigma_{5} ; \tau_{0}, \tau_{1}, \tau_{2}\right) \mapsto\left(\tau_{0}, \tau_{1}, \sigma_{0}, \sigma_{5}, \sigma_{3}, \tau_{2}\right)=:\left(\rho_{0}, \ldots, \rho_{5}\right)
$$

on $W(\mathcal{D})$ defines the polytope $\{3,4,3,3,4\}$ with group

$$
\left\langle\rho_{0}, \ldots, \rho_{5}\right\rangle=[3,4,3,3,4]=W(\mathcal{D}) \ltimes\left(S_{3} \times C_{2}\right) .
$$

It follows from these observations that the Coxeter group [3, 4, 3, 3, 3] has subgroups $[3,3,4,3,3]$ and $[3,4,3,3,4]$ of indices 5 and 10 , respectively. This fact can also be proved by simplex dissection of hyperbolic simplices.

Note that, with $\mathcal{L}=\{3,3,3\}$ and $\mathcal{K}=\{3\}$ (on 3 vertices), we also have

$$
\{3,3,3,4,3\}=\{3,3,3\}^{\{3\}}, \quad[3,3,3,4,3]=W(\mathcal{D}) \ltimes S_{3},
$$

with diagram

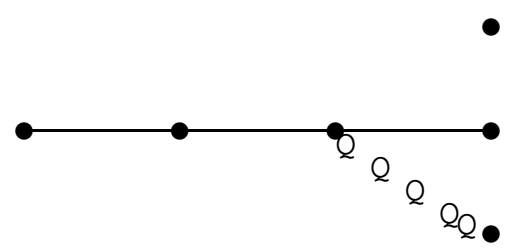

and the same group as in the first example is now expressed in a different way.

\section{A UniVERSALity PROPERTY OF $2^{\mathcal{K}, \mathcal{G}}$}

In this section, we prove a property of certain polytopes $2^{\mathcal{K}, \mathcal{G}}$ and $\mathcal{L}^{\mathcal{K}, \mathcal{G}}$, which allows us to identify them as universal polytopes in their classes. The crucial concept is that of an extension of a given diagram to a diagram on a larger set of nodes, which preserves the action of $\mathcal{K}$. It is striking that this method enables us to deal with many classes of locally toroidal regular polytopes, and to find the finite universal polytopes in these classes. This also lays the foundations for Sections 6 and 7 .

Let $\mathcal{K}$ be a regular $n$-polytope with group $A(\mathcal{K})=\left\langle\tau_{0}, \ldots, \tau_{n-1}\right\rangle$ and (basic) facet $\mathcal{F}:=\mathcal{K}_{n-1}$; then $A(\mathcal{F})=\left\langle\tau_{0}, \ldots, \tau_{n-2}\right\rangle$. Let $\mathcal{H}$ be an $\mathcal{F}$-admissible diagram on the vertex set $V(\mathcal{F})$ of $\mathcal{F}$, with the base vertex $F_{0}$ of $\mathcal{K}$ as initial vertex. Then the regular $n$-polytope $2^{\mathcal{F}, \mathcal{H}}$ is defined and has vertex-figures isomorphic to $\mathcal{F}$. Now, if $\mathcal{G}$ is any $\mathcal{K}$-admissible diagram on the vertex set $V(\mathcal{K})$ of $\mathcal{K}$ such that $\mathcal{H}$ is the induced subdiagram of $\mathcal{G}$ on $V(\mathcal{F})$, then $2^{\mathcal{K}, \mathcal{G}}$ is a regular $(n+1)$-polytope in the class $\left\langle 2^{\mathcal{F}, \mathcal{H}}, \mathcal{K}\right\rangle$. However, given $\mathcal{H}$, such a diagram $\mathcal{G}$ need not exist in general. If $\mathcal{G}$ does exist, then we call $\mathcal{H}$ a $\mathcal{K}$-extendable diagram, and $\mathcal{G}$ a $\mathcal{K}$-extension of $\mathcal{H}$. 
For instance, let $\mathcal{K}$ be the 24 -cell $\{3,4,3\}$ (with 24 octahedral facets), and $\mathcal{H}$ the diagram on the octahedron $\{3,4\}$ which connects antipodal vertices by a branch labelled $s$. Then we can take for $\mathcal{G}$ the diagram on the 24 vertices of $\mathcal{K}$ whose restriction to the vertex-set of each facet is $\mathcal{H}$. However, we could also add further branches in a suitable way.

To give an example of a diagram $\mathcal{H}$ which is not $\mathcal{K}$-extendable, choose for $\mathcal{K}$ the hemi-cube $\{4,3\}_{3}$ (obtained by identifying antipodal vertices of the 3 -cube), and for $\mathcal{H}$ the diagram on the four vertices of $\mathcal{F}:=\{4\}$ which connects antipodal vertices of $\mathcal{F}$ by a branch with a label at least 3 , but connects no other vertices of $\mathcal{F}$. Then $\mathcal{H}$ is not $\mathcal{K}$-extendable, because in $\mathcal{K}$ antipodal vertices of $\mathcal{F}$ are connected by edges of $\mathcal{K}$.

It is easy to see that a diagram $\mathcal{H}$ is $\mathcal{K}$-extendable if and only if it has the following property:

$$
\left\{\begin{array}{l}
\text { If two diagonals (pairs of vertices) of } \mathcal{F} \text { are equivalent under } \\
A(\mathcal{K}) \text {, then the corresponding branches in } \mathcal{H} \text { have the same } \\
\text { label (possibly 2). }
\end{array}\right.
$$

Note that it is not required that the two diagonals be equivalent under $A(\mathcal{F})$. Given a $\mathcal{K}$-extendable diagram $\mathcal{H}$, there exists a universal $\mathcal{K}$-extension $\mathcal{D}$ of $\mathcal{H}$, in the sense that for any $\mathcal{K}$-extension $\mathcal{G}$ there is a homomorphism $W(\mathcal{D}) \mapsto W(\mathcal{G})$ between the corresponding Coxeter groups, which maps generators onto corresponding generators. This diagram $\mathcal{D}$ with node set $V(\mathcal{K})$ is obtained as follows.

First, take the branches and labels of $\mathcal{H}$ and all their transforms under elements of $A(\mathcal{K})$; by (10), this already gives a $\mathcal{K}$-extension. (This corresponds to the above example for the 24-cell.) Second, complete the diagram $\mathcal{D}$ by adding (if possible) branches with label $\infty$, one for each diagonal of $\mathcal{K}$ which is not equivalent under $A(\mathcal{K})$ to a diagonal of $\mathcal{F}$. This gives a $\mathcal{K}$-extension of $\mathcal{H}$. Note that, in constructing the universal $\mathcal{K}$-extension, we have added as many branches with marks $\infty$ as possible, while preserving the $\mathcal{K}$-extension property. Note also that a $\mathcal{K}$-extendable diagram $\mathcal{H}$ coincides with all its $\mathcal{K}$-extensions if and only if $\mathcal{K}$ is flat. The following theorem shows the significance of the universal $\mathcal{K}$-extension $\mathcal{D}$.

Theorem 5.1. Let $\mathcal{K}$ be a regular $n$-polytope with facet $\mathcal{F}$, and let $\mathcal{H}$ be an $\mathcal{F}$ admissible diagram on the vertex-set of $\mathcal{F}$ which is $\mathcal{K}$-extendable. Then the universal polytope $\left\{2^{\mathcal{F}, \mathcal{H}}, \mathcal{K}\right\}$ exists, and

$$
\left\{2^{\mathcal{F}, \mathcal{H}}, \mathcal{K}\right\}=2^{\mathcal{K}, \mathcal{D}},
$$

where $\mathcal{D}$ is the universal $\mathcal{K}$-extension of $\mathcal{H}$.

Proof. By construction, $2^{\mathcal{K}, \mathcal{D}}$ is in $\left\langle 2^{\mathcal{F}, \mathcal{H}}, \mathcal{K}\right\rangle$, so that the universal polytope does indeed exist. It remains to prove that $2^{\mathcal{K}, \mathcal{D}}$ is itself universal.

Let $\mathcal{P}:=\left\{2^{\mathcal{F}, \mathcal{H}}, \mathcal{K}\right\}$ and $A(\mathcal{P})=:\left\langle\alpha_{0}, \ldots, \alpha_{n}\right\rangle$. If $N_{0}$ denotes the normal closure of $\alpha_{0}$ in $A(\mathcal{P})$, then

$$
A(\mathcal{P})=N_{0} \cdot\left\langle\alpha_{1}, \ldots, \alpha_{n}\right\rangle\left(=N_{0} \cdot A(\mathcal{K})\right)
$$

For the polytope $2^{\mathcal{K}, \mathcal{D}}$, the normal closure of $\rho_{0}=\sigma_{0}$ in $A\left(2^{\mathcal{K}, \mathcal{D}}\right)=\left\langle\rho_{0}, \ldots, \rho_{n}\right\rangle$ is the subgroup $W(\mathcal{D})=\left\langle\sigma_{i} \mid i \in V(\mathcal{K})\right\rangle$; here 0 is the base vertex of $\mathcal{K}$. This shows that

$$
A\left(2^{\mathcal{K}, \mathcal{D}}\right)=W(\mathcal{D}) \cdot\left\langle\rho_{1}, \ldots, \rho_{n}\right\rangle \simeq W(\mathcal{D}) \ltimes A(\mathcal{K}) .
$$


On the other hand, the mapping $\alpha_{i} \mapsto \rho_{i}(i=0, \ldots, n)$ defines a surjective homomorphism $f: A(\mathcal{P}) \mapsto A\left(2^{\mathcal{K}, \mathcal{D}}\right)$, which is one-to-one on $\left\langle\alpha_{1}, \ldots, \alpha_{n}\right\rangle$, and maps $N_{0}$ onto $W(\mathcal{D})$. It follows that the product in (11) is semi-direct.

Next, we observe that the action of $A(\mathcal{K})$ on $N_{0}$ is equivalent to that on $W(\mathcal{D})$. More precisely, if $\varphi, \psi \in\left\langle\alpha_{1}, \ldots, \alpha_{n}\right\rangle$, then

$$
\begin{aligned}
\varphi \alpha_{0} \varphi^{-1}=\psi \alpha_{0} \psi^{-1} & \Longleftrightarrow f(\varphi) \sigma_{0} f(\varphi)^{-1}=f(\psi) \sigma_{0} f(\psi)^{-1} \\
& (\Longleftrightarrow f(\varphi)(0)=f(\psi)(0), \text { as vertices of } \mathcal{K}) .
\end{aligned}
$$

To prove the less obvious assertion, note that $f\left(\psi^{-1} \varphi\right) \sigma_{0} f\left(\psi^{-1} \varphi\right)^{-1}=\sigma_{0}$ from the second equation, and thus $f\left(\psi^{-1} \varphi\right)(0)=0$. But then $f\left(\psi^{-1} \varphi\right) \in\left\langle\rho_{2}, \ldots, \rho_{n}\right\rangle$, and hence $\psi^{-1} \varphi \in\left\langle\alpha_{2}, \ldots, \alpha_{n}\right\rangle$, because $f$ is one-to-one on $\left\langle\alpha_{1}, \ldots, \alpha_{n}\right\rangle$.

Now by (13) the generators of $N_{0}$ are in one-to-one correspondence with the vertices of $\mathcal{K}$. For $i \in V(\mathcal{K})$, define $\beta_{i}:=\varphi_{i} \alpha_{0} \varphi_{i}^{-1}$, where $\varphi_{i} \in\left\langle\alpha_{1}, \ldots, \alpha_{n}\right\rangle$ is such that $f\left(\varphi_{i}\right)(0)=i$. Then $N_{0}=\left\langle\beta_{i} \mid i \in V(\mathcal{K})\right\rangle, \beta_{0}=\alpha_{0}$, and $f\left(\beta_{i}\right)=\sigma_{i}$ for all $i \in V(\mathcal{K})$. To prove that $f$ is also one-to-one on $N_{0}$, it suffices to check that $N_{0}$ belongs to the same diagram $\mathcal{D}$ as $W(\mathcal{D})$. Two cases have to be considered.

First, consider $\beta_{i} \beta_{j}$, with $\{i, j\}$ representing a diagonal class of $\mathcal{K}$ which is equivalent under $A(\mathcal{K})$ to a diagonal class of $\mathcal{F}$. Then we may assume that $i, j \in V(\mathcal{F})$. Since $\mathcal{P}$ and $2^{\mathcal{K}, \mathcal{D}}$ have the same facets $2^{\mathcal{F}, \mathcal{H}}$, we know that $f$ must be one-to-one on the subgroup $\left\langle\beta_{k} \mid k \in V(\mathcal{F})\right\rangle$. It follows that $\beta_{i} \beta_{j}$ and $\sigma_{i} \sigma_{j}$ have the same order.

Second, if $\{i, j\}$ represents a diagonal class of $\mathcal{K}$ which is not equivalent under $A(\mathcal{K})$ to one of $\mathcal{F}$, then since $\mathcal{D}$ is universal, $\sigma_{i} \sigma_{j}$ already has infinite order, and so does $\beta_{i} \beta_{j}$.

It follows that $f$ is one-to-one on $A(\mathcal{P})$ and thus $\mathcal{P} \simeq 2^{\mathcal{K}, \mathcal{D}}$, which completes the proof.

Call a polytope $\mathcal{P}$ weakly neighbourly if any two vertices of $\mathcal{P}$ lie in a common facet. Examples of such polytopes are the toroids $\{4,4\}_{(3,0)}$ and $\{3,3,4,3\}_{(2,0,0,0)}$; see Section 3.

Corollary 5.2. Let $\mathcal{K}$ be a regular n-polytope with facet $\mathcal{F}$, and let $\mathcal{D}$ be the universal $\mathcal{K}$-extension of the trivial diagram on $\mathcal{F}$. Then $\left\{2^{\mathcal{F}}, \mathcal{K}\right\}$ exists and coincides with $2^{\mathcal{K}, \mathcal{D}}$. In particular, $\left\{2^{\mathcal{F}}, \mathcal{K}\right\}$ is finite (with group $C_{2}^{|V(\mathcal{K})|} \ltimes A(\mathcal{K})$ ) if and only if $\mathcal{K}$ is finite and weakly neighbourly.

Proof. Apply Theorem 5.1 with $\mathcal{H}$ the trivial diagram on $\mathcal{F}$. Then $\mathcal{H}$ is $\mathcal{K}$ extendable by the trivial diagram on $\mathcal{K}$. From the definition of $\mathcal{D}$, the polytope $2^{\mathcal{K}, \mathcal{D}}$ is finite if and only if $\mathcal{K}$ is finite, and each pair of vertices of $\mathcal{K}$ is equivalent under $A(\mathcal{K})$ to a pair of vertices of $\mathcal{F}$; that is, if and only if $\mathcal{K}$ is finite and weakly neighbourly; in this case, $W(\mathcal{D}) \simeq C_{2}^{|V(\mathcal{K})|}$ because $\mathcal{D}$ is trivial.

For the next example, recall that a polytope is called simplicial if all its facets are isomorphic to simplices, and cubical if its facets are isomorphic to cubes.

Corollary 5.3. Let $\mathcal{K}$ be a simplicial regular $n$-polytope, and let $\mathcal{D}$ be the universal $\mathcal{K}$-extension of the trivial diagram on its facet $\mathcal{F}$. Then the cubical regular $(n+1)$-polytope $\left\{\left\{4,3^{n-2}\right\}, \mathcal{K}\right\}$ exists, and coincides with $2^{\mathcal{K}, \mathcal{D}}$. In particular, $\left\{\left\{4,3^{n-2}\right\}, \mathcal{K}\right\}$ is finite (and then equal to $2^{\mathcal{K}}$, with group the semidirect product $\left.C_{2}^{|V(\mathcal{K})|} \ltimes A(\mathcal{K})\right)$ if and only if $\mathcal{K}$ is finite and neighbourly. 
Proof. Apply Corollary 5.2 with $\mathcal{F}=\left\{3^{n-2}\right\}$, and use $2^{\mathcal{F}}=\left\{4,3^{n-2}\right\}$. Further, observe that a simplicial polytope is neighbourly if and only if it is weakly neighbourly.

Corollary 5.3 says that the only finite universal cubical regular polytopes are those in which the vertex-figure is finite and neighbourly. It would therefore be interesting to be able to characterize the finite neighbourly regular polytopes.

We proceed with further applications of Theorem 5.1. In all these cases, $\mathcal{K}$ will be a regular $n$-polytope with centrally symmetric facets $\mathcal{F}$, where we call a regular polytope $\mathcal{P}$ centrally symmetric if its group $A(\mathcal{P})$ contains a proper central involution, which does not fix any of its vertices. (Note that, if a central involution in $A(\mathcal{P})$ fixes one vertex, then it fixes every vertex, and thus acts on the vertexset of $\mathcal{P}$ like the identity. It is therefore natural to leave such involutions out of consideration.) A proper central involution in $A(\mathcal{P})$ thus pairs up antipodal vertices of $\mathcal{P}$. Note that a central involution in the group $A(\mathcal{P})$ of a regular polytope $\mathcal{P}$ whose faces are uniquely determined by their vertex-sets (as is the case, for example, when $\mathcal{P}$ is a lattice) must be proper, and so makes $\mathcal{P}$ centrally symmetric.

Antipodal vertices of a centrally symmetric regular $k$-polytope $\mathcal{P}$ cannot be joined by an edge unless $\mathcal{P}$ has only two vertices, or, equivalently, $p_{1}=2$ in the Schläfli symbol $\left\{p_{1}, \ldots, p_{k-1}\right\}$ of $\mathcal{P}$. To see this, let $A(\mathcal{P})=\left\langle\rho_{0}, \ldots, \rho_{k-1}\right\rangle$, with the $\rho_{i}$ the distinguished generators with respect to the base flag $\left\{F_{0}, \ldots, F_{k-1}\right\}$ of $\mathcal{P}$, and let $\alpha \in A(\mathcal{P})$ be a proper central involution. If the vertices $F_{0}$ and $\rho_{0}\left(F_{0}\right)$ of the base edge $F_{1}$ are antipodal, then $\alpha\left(F_{0}\right)=\rho_{0}\left(F_{0}\right)$ implies that $\rho_{0} \in \alpha\left\langle\rho_{1}, \ldots, \rho_{k-1}\right\rangle$, and hence that $A(\mathcal{P}) \cong\left\langle\rho_{1}, \ldots, \rho_{k-1}\right\rangle \times C_{2}$. It follows that $\mathcal{P}$ has only two vertices, as claimed.

Now let $\mathcal{K}$ and $\mathcal{F}$ be as before, and suppose that $\mathcal{F}$ is centrally symmetric. We write $\mathcal{H}=\mathcal{H}_{s}(\mathcal{F})$ for the diagram on the vertex-set $V(\mathcal{F})$ of $\mathcal{F}$ which connects antipodal vertices of $\mathcal{F}$ by a branch marked $s(\geq 2)$. In the cases we consider, $\mathcal{H}_{s}(\mathcal{F})$ will be $\mathcal{K}$-extendable, and we denote its universal $\mathcal{K}$-extension by $\mathcal{D}:=\mathcal{D}_{s}(\mathcal{K})$. Note that, if $\mathcal{F}=\left\{3^{n-3}, 4\right\}$, then

$$
2^{\mathcal{F}, \mathcal{H}_{s}(\mathcal{F})}=\left\{4,3^{n-3}, 4\right\}_{\left(2 s, 0^{n-2}\right)},
$$

a toroid with group $\left[4,3^{n-3}, 4\right]_{\left(2 s, 0^{n-2}\right)}=D_{s}^{n-1} \ltimes\left[3^{n-3}, 4\right]$. If $\mathcal{F}=\{3,4\}$, then $\mathcal{H}_{s}(\mathcal{F})$ is the diagram which connects antipodal vertices of the octahedron by a branch marked $s$, and $2^{\mathcal{F}, \mathcal{H}_{s}(\mathcal{F})}$ is the toroid $\{4,3,4\}_{(2 s, 0,0)}$ obtained from a $(2 s \times$ $2 s \times 2 s$ ) cubical grid by identifying opposite walls.

Corollary 5.4. Let $s \geq 2$, and let $\mathcal{K}:=\{4,4\}_{(b, c)}$ with $b \geq 2$ and $c=0$ or $b$. Then

$$
\left\{\{4,4\}_{(2 s, 0)}, \mathcal{K}\right\}=2^{\mathcal{K}, \mathcal{D}_{s}(\mathcal{K})},
$$

which is finite if and only if $s \geq 2$ and $(b, c)=(2,0)$ or $s=2$ and $(b, c)=(3,0)$. In the finite cases, the groups are $\left(D_{s} \times D_{s}\right) \ltimes[4,4]_{(2,0)}$ and $C_{2}^{9} \ltimes[4,4]_{(3,0)}$, respectively.

Proof. Here, $\mathcal{F}=\{4\}$, and $\mathcal{H}=\mathcal{H}_{s}(\mathcal{F})$ is $\mathcal{K}$-extendable for all $(b, c)$. The facets are $2^{\mathcal{F}, \mathcal{H}}=\{4,4\}_{(2 s, 0)}$. The group of $2^{\mathcal{K}, \mathcal{D}}$ is finite if and only if $W(\mathcal{D})$ is finite, that is, if and only if $s \geq 2$ and $(b, c)=(2,0)$, or $s=2$ and $(b, c)=(3,0)$. In the finite cases, $W(\mathcal{D})=D_{s} \times D_{s}$ or $W(\mathcal{D})=C_{2}^{9}$, respectively, so that the groups are as described.

For another construction of the polytopes in Corollary 5.4, the reader is referred to [19]. The structure of the polytopes for $s=1$ will be discussed in Section 7 . 
Corollary 5.5. Let $s \geq 2$, and let $\mathcal{K}:=\{3,4,3\}$. Then

$$
\left\{\{4,3,4\}_{(2 s, 0,0)}, \mathcal{K}\right\}=2^{\mathcal{K}, \mathcal{D}_{s}(\mathcal{K})},
$$

which is infinite for all $s$.

Proof. Now $\mathcal{F}=\{3,4\}$, and again $\mathcal{H}=\mathcal{H}_{s}(\mathcal{F})$ is $\mathcal{K}$-extendable. The facets are $\{4,3,4\}_{(2 s, 0,0)}$, and the group $W(\mathcal{D})$ is infinite for all $s \geq 2$.

Corollary 5.6. Let $s, t \geq 2, k=1$ or 2 , and let $\mathcal{K}:=\{3,3,4,3\}_{\left(t^{k}, 0^{4-k}\right)}$. Then

$$
\left\{\{4,3,3,4\}_{(2 s, 0,0,0)}, \mathcal{K}\right\}=2^{\mathcal{K}, \mathcal{D}_{s}(\mathcal{K})},
$$

which is an infinite polytope unless $(s, t, k)=(2,2,1)$. If $(s, t, k)=(2,2,1)$, the group of the polytope is $C_{2}^{16} \ltimes[3,3,4,3]_{(2,0,0,0)}$, of order 1207959552 .

Proof. We now have $\mathcal{F}=\{3,3,4\}$, the 4-crosspolytope. Note that antipodal vertices of facets of $\mathcal{K}$ are never joined by an edge, so that $\mathcal{H}=\mathcal{H}_{s}(\mathcal{F})$ is always $\mathcal{K}$-extendable. Observe also that, if $(t, k)=(2,1)$ or $(2,2)$, then in $\mathcal{K}$ two vertices can be antipodal vertices of more than one facet (in fact, eight facets); hence, if we generate a $\mathcal{K}$-extension by applying all automorphisms of $\mathcal{K}$ to $\mathcal{H}$, we can only take one branch for each such pair of vertices. Now the facets are isomorphic to $\{4,3,3,4\}_{(2 s, 0,0,0)}$. The group of the polytope is infinite unless $\mathcal{K}$ is weakly neighbourly and $s=2$. This leaves the exceptional case $(s, t, k)=(2,2,1)$; here, $\mathcal{D}_{2}(\mathcal{K})$ pairs up the 16 vertices of $\mathcal{K}$ to give a group $C_{2}^{16} \ltimes[3,3,4,3]_{(2,0,0,0)}$. (When $\mathcal{K}=\{3,3,4,3\}_{(2,0,0,0)}$, the diagram $\mathcal{D}_{s}(\mathcal{K})$ splits into four components, each given by a complete graph on 4 nodes with all branches marked $s$.)

Our next result generalizes Theorem 5.1, and deals with the polytopes $\mathcal{L}^{\mathcal{K}, \mathcal{G}}$ for universal polytopes $\mathcal{L}$ of rank at least 2. Here we do not need the concept of a $\mathcal{K}$-extension of a diagram.

Theorem 5.7. Let $\mathcal{K}$ be a regular n-polytope with facet $\mathcal{F}$, let $\mathcal{G}$ be a $\mathcal{K}$-admissible diagram on the vertex-set of $\mathcal{K}$, and let $\mathcal{H}$ denote the induced subdiagram of $\mathcal{G}$ on the vertex-set of $\mathcal{F}$. Let $m \geq 2$, and let $\mathcal{L}:=\left\{q_{1}, \ldots, q_{m-1}\right\}$ and $\mathcal{L}_{0}:=\left\{q_{2}, \ldots, q_{m-1}\right\}$, the vertex-figure of $\mathcal{L}$. Then the universal regular $(n+m)$-polytope $\left\{\mathcal{L}^{\mathcal{F}, \mathcal{H}}, \mathcal{L}_{0}^{\mathcal{K}}, \mathcal{G}\right\}$ exists, and

$$
\left\{\mathcal{L}^{\mathcal{F}, \mathcal{H}}, \mathcal{L}_{0}^{\mathcal{K}, \mathcal{G}}\right\}=\mathcal{L}^{\mathcal{K}, \mathcal{G}}
$$

Proof. The proof is similar to that of Theorem 5.1. Let $\mathcal{P}:=\left\{\mathcal{L}^{\mathcal{F}, \mathcal{H}}, \mathcal{L}_{0}^{\mathcal{K}, \mathcal{G}}\right\}$. We choose $N_{0}$ to be the normal closure of $\left\langle\alpha_{0}, \ldots, \alpha_{m-1}\right\rangle$ in $A(\mathcal{P}):=\left\langle\alpha_{0}, \ldots, \alpha_{m+n-1}\right\rangle$, and conclude as before that

$$
A(\mathcal{P}) \simeq N_{0} \ltimes\left\langle\alpha_{m}, \ldots, \alpha_{m+n-1}\right\rangle \simeq N_{0} \ltimes A(\mathcal{K}) .
$$

Then an analogue of (13) holds with the suffix 0 replaced by $m-1$; the corresponding statement is also true for the suffixes $0,1, \ldots, m-2$, but here it is trivial because $\varphi, \psi \in\left\langle\alpha_{m}, \ldots, \alpha_{m+n-1}\right\rangle$ commute with each $\alpha_{j}$ with $j \leq m-2$. It follows that $N_{0}=\left\langle\beta_{i} \mid i \in V(\mathcal{D})\right\rangle$, with $\mathcal{D}$ the diagram used to define $\mathcal{L}^{\mathcal{K}, \mathcal{G}}$ (see Section 4). Again it can be proved that $N_{0}$ belongs to the same diagram $\mathcal{D}$, because the polytopes have the same facets and the same vertex-figures. The details are left to the reader.

Corollary 5.8. Let $\mathcal{K}:=\{3,4\}$, and let $\mathcal{G}_{s}(\mathcal{K})$ be the diagram on the vertex-set of $\mathcal{K}$ connecting antipodal vertices by a branch labelled $s \geq 2$. Then

$$
\left\{\{3,4,3\},\{4,3,4\}_{(2 s, 0,0)}\right\}=\{3\}^{\{3,4\}, \mathcal{G}_{s}(\mathcal{K})},
$$


Proof. In this case, the facets are $\{3\}^{\{3\}}=\{3,4,3\}$ and the vertex-figures are $2^{\mathcal{K}, \mathcal{G}_{s}(\mathcal{K})}=\{4,3,4\}_{(2 s, 0,0)}$. Note that Corollary 5.8 gives another construction of the duals of the 5-polytopes occurring in Corollary 5.5. If the vertices of $\mathcal{K}=\{3,4\}$ are labelled $1, \ldots, 6$, with antipodes labelled $i, i+3(\bmod 6)$, then the diagram $\mathcal{D}$ involved in the construction of the polytopes of Corollary 5.8 is the diagram which we discuss further in the next section.

We conclude this section with yet another application of our methods to an interesting extension problem for regular polytopes. Here we restrict ourselves to the most important case where all the entries in the Schläfli symbols are at least 3; the general case can easily be derived from this.

Theorem 5.9. Let $n \geq 2$, and let $\mathcal{K}$ be a regular $n$-polytope of type $\left\{p_{1}, \ldots, p_{n-1}\right\}$ with $p_{1}, \ldots, p_{n-1} \geq 3$. Let $r \geq 2, m \geq 1$ and $q_{1}, \ldots, q_{m-1} \geq 3$. Then there exists a regular $(m+n)$-polytope $\mathcal{P}$ with the following properties:

(a) The polytope is of type $\left\{q_{1}, \ldots, q_{m-1}, 2 r, p_{1}, \ldots, p_{n-1}\right\}$, and its co-faces to $(m-1)$-faces are isomorphic to $\mathcal{K}$.

(b) $\mathcal{P}$ is "universal" among all regular $(m+n)$-polytopes which have the property of part (a); that is, any other such regular $(m+n)$-polytope is a quotient of $\mathcal{P}$.

(c) $\mathcal{P}$ is finite if and only if $m=1, r=2$ and $\mathcal{K}$ is finite and neighbourly, or $m=2, p_{1}=3, r=2$ and $\mathcal{K}=\{3\}$. In these cases, $\mathcal{P}=2^{\mathcal{K}}$ or $\mathcal{P}=\{3,4,3\}$, with groups $C_{2}^{|V(\mathcal{K})|} \ltimes A(\mathcal{K})$ or $[3,4,3]$, respectively.

(d) $\mathcal{P}=\left\{q_{1}, \ldots, q_{m-1}\right\}^{\mathcal{K}, \mathcal{G}}$, where $\mathcal{G}$ is the complete graph on the vertex set of $\mathcal{K}$, in which the branches representing edges of $\mathcal{K}$ are marked $r$, while all other branches are marked $\infty$. In particular, the $m$-faces of $\mathcal{P}$ are isomorphic to $\left\{q_{1}, \ldots, q_{m-1}\right\}$.

Proof. We use the same method of proof as for Theorems 5.1 and 5.7. Let $\mathcal{L}:=$ $\left\{q_{1}, \ldots, q_{m-1}\right\}$, and let $\mathcal{G}$ be as in $(\mathrm{d})$. First note that $\mathcal{L}^{\mathcal{K}, \mathcal{G}}$ (with group $A\left(\mathcal{L}^{\mathcal{K}, \mathcal{G}}\right)=$ $\left.\left\langle\rho_{0}, \ldots, \rho_{m+n-1}\right\rangle\right)$ is a polytope which satisfies (a). On the other hand, the existence of any regular polytope with property (a) implies the existence of a universal such polytope; a proof of this fact can be given by adapting the proof of Theorem 1 in [26] to the situation discussed here. Let $\mathcal{P}$ be this universal polytope, and let $A(\mathcal{P})=\left\langle\alpha_{0}, \ldots, \alpha_{m+n-1}\right\rangle$. As in the proof of Theorem 5.7, if $N_{0}$ denotes the normal closure of $\left\langle\alpha_{0}, \ldots, \alpha_{m-1}\right\rangle$ in $A(\mathcal{P})$, then (15) holds and we again arrive at $N_{0}=\left\langle\beta_{i} \mid i \in V(\mathcal{D})\right\rangle$, with $\mathcal{D}$ the diagram used to define $\mathcal{L}^{\mathcal{K}, \mathcal{G}}$. Here, $\beta_{i}=\alpha_{i}$ if $i \leq m-2$, or is the conjugate of $\alpha_{m-1}$ which corresponds to the vertex $i$ of $V(\mathcal{K})$ otherwise. Again we need to check that $N_{0}$ belongs to the same diagram $\mathcal{D}$ as the group $W(\mathcal{D})=\left\langle\sigma_{i} \mid i \in V(\mathcal{D})\right\rangle$, that is, that $\beta_{i} \beta_{j}$ and $\sigma_{i} \sigma_{j}$ have the same order for any two nodes $i, j$ of $\mathcal{D}$.

If $i, j \leq m-1$, then $\beta_{i} \beta_{j}=\alpha_{i} \alpha_{j}$ and $\sigma_{i} \sigma_{j}=\rho_{i} \rho_{j}$ clearly have the same order, because $\mathcal{P}$ and $\mathcal{L}^{\mathcal{K}, \mathcal{G}}$ have the same Schläfli symbols. Since $A(\mathcal{K})=\left\langle\alpha_{m}, \ldots, \alpha_{m+n-1}\right\rangle$ acts on $N_{0}$ as it does on $W(\mathcal{D})$, the elements $\beta_{i} \beta_{j}$ and $\sigma_{i} \sigma_{j}$ are conjugate to $\beta_{i} \beta_{m-1}$ and $\sigma_{i} \sigma_{m-1}$, respectively, if $i \leq m-2$ and $j \in V(\mathcal{K})$; hence the orders are again the same.

Now let $i, j \in V(\mathcal{K})$. If $i, j$ are joined by an edge of $\mathcal{K}$, then modulo $A(\mathcal{K})$ we may assume that $i=m-1=F_{0}$, the base vertex of $\mathcal{K}$, and $j=\rho_{m}\left(F_{0}\right)\left(=\alpha_{m}\left(F_{0}\right)\right)$, the other vertex in the base edge of $\mathcal{K}$. But then 


$$
\sigma_{i} \sigma_{j}=\sigma_{F_{0}} \rho_{m} \sigma_{F_{0}} \rho_{m}=\left(\sigma_{F_{0}} \rho_{m}\right)^{2}=\left(\rho_{m-1} \rho_{m}\right)^{2},
$$

and similarly

$$
\beta_{i} \beta_{j}=\beta_{F_{0}} \alpha_{m} \beta_{F_{0}} \alpha_{m}=\left(\beta_{F_{0}} \alpha_{m}\right)^{2}=\left(\alpha_{m-1} \alpha_{m}\right)^{2}
$$

It follows that both elements have order $2 r$, because the corresponding entry in the Schläfli symbol is $2 r$. Finally, if $i, j$ are not joined by an edge of $\mathcal{K}$, then by the construction of $\mathcal{G}$ the order of $\sigma_{i} \sigma_{j}$ is infinite, and so is the order of $\beta_{i} \beta_{j}$.

It follows that the homomorphism $A(\mathcal{P}) \mapsto A\left(\mathcal{L}^{\mathcal{K}, \mathcal{G}}\right)$ is an isomorphism, and thus $\mathcal{P}$ and $\mathcal{L}^{\mathcal{K}, \mathcal{G}}$ are isomorphic polytopes. This proves parts (a), (b) and (d) of the theorem.

Furthermore, $\mathcal{L}^{\mathcal{K}, \mathcal{G}}$ is finite if and only if $W(\mathcal{D})$ and $A(\mathcal{K})$ are finite. Let $\mathcal{K}$ be finite. If $m=1$, then $W(\mathcal{D})$ is finite if and only if $\mathcal{G}$ has no branches marked $r \geq 3$ or $\infty$; that is, if and only if $\mathcal{K}$ is neighbourly and $r=2$. In this case, $\mathcal{P}=2^{\mathcal{K}}$, $W(\mathcal{D}) \cong C_{2}^{|V(\mathcal{K})|}$, and $A(\mathcal{P}) \cong C_{2}^{|V(\mathcal{K})|} \ltimes A(\mathcal{K})$. If $m \geq 2$, then $W(\mathcal{D})$ can only be finite if $r=2$, and the node $m-2$ of $\mathcal{D}$ is contained in at most 3 branches (marked $q_{m-2} \geq 3$ or $q_{m-1} \geq 3$ ); that is, if and only if $r=2$ and $\mathcal{K}=\mathcal{L}=\{3\}$ (and $m=n=2)$. But if $r=2$ and $\mathcal{K}=\mathcal{L}=\{3\}$, then $\mathcal{P}=\{3,4,3\}$. This completes the proof.

\section{The polytopes $\left\{\{3,4,3\},\{4,3,4\}_{\mathbf{s}}\right\}$}

As was remarked in the Introduction, up to duality there is only one possible Schläfli symbol for locally toroidal regular polytopes of rank 5 , namely $\{3,4,3,4\}$. The polytopes are now members of a class $\left\langle\{3,4,3\},\{4,3,4\}_{\mathbf{s}}\right\rangle$, for some $\mathbf{s}=$ $\left(s^{k}, 0^{3-k}\right)$ with $s \geq 2$ and $k=1,2,3$. It is known that the corresponding universal polytopes $\mathcal{P}_{\mathbf{s}}:=\left\{\{3,4,3\},\{4,3,4\}_{\mathbf{s}}\right\}$ exist for all $\mathbf{s}$, but are finite only if $\mathbf{s}=(2,0,0),(2,2,0)$ or $(2,2,2)([21])$. However, the method of proof in [21] is constructive only for $k=1$. If $k=1$ and $s \geq 2$, the corresponding polytope $\mathcal{P}_{(s, 0,0)}$ can be obtained from the Coxeter group $U_{s}$ with diagram
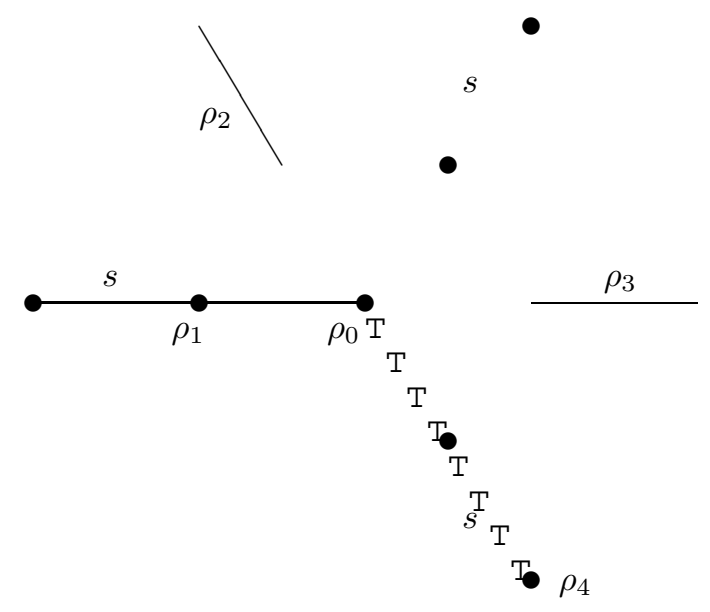
by choosing the generators $\rho_{0}, \ldots, \rho_{4}$ of $A\left(\mathcal{P}_{(s, 0,0)}\right)=U_{s} \ltimes S_{3}$ as indicated ( $\rho_{2}$ and $\rho_{3}$ are diagram automorphisms).

The main result of this section, Theorem 6.3, uses the geometry of the octahedron to obtain, for each $k=1,2,3$ and each odd $\mathbf{s}=\left(s^{k}, 0^{3-k}\right)$ (meaning that $s$ is odd), an infinite polytope in $\left\langle\{3,4,3\},\{4,3,4\}_{\mathbf{s}}\right\rangle$. This is based on Corollary 5.8 of the previous section, and gives a more constructive way of proving the non-finiteness of the corresponding polytope $\mathcal{P}_{\mathbf{s}}$. If $\mathbf{s}=(s, 0,0)$, the construction gives $\mathcal{P}_{\mathbf{s}}$ itself.

It is interesting to note that the groups of these polytopes are residually finite, because they admit faithful representations as linear groups over the real field (Malcev [11]). Recall that a group $U$ is residually finite if, for each finite subset $\left\{\varphi_{1}, \ldots, \varphi_{m}\right\}$ of $U \backslash\{\varepsilon\}$, there exists a homomorphism $f$ of $U$ onto some finite group, such that $f\left(\varphi_{j}\right) \neq \varepsilon$ for each $j=1, \ldots, m$. It was proved in [20] that, if a class $\left\langle\mathcal{P}_{1}, \mathcal{P}_{2}\right\rangle$ contains an infinite polytope $\mathcal{P}$ with a residually finite group, then it contains infinitely many regular polytopes which are finite and are covered by $\mathcal{P}$. In particular, the construction of such infinite polytopes implies that all classes $\left\langle\{3,4,3\},\{4,3,4\}_{\mathbf{s}}\right\rangle$ with s odd contain infinitely many finite regular polytopes. We conjecture this result to be true for even $\mathbf{s}$ with $s>2$ as well. Note that there are also similar results for each class which contains an infinite polytope $2^{\mathcal{K}, \mathcal{G}}$ or $\mathcal{L}^{\mathcal{K}, \mathcal{G}}$ with $\mathcal{K}$ finite.

In our construction, we shall frequently use the following quotient relations among the polytopes $\mathcal{P}_{\mathbf{s}}([21])$ :

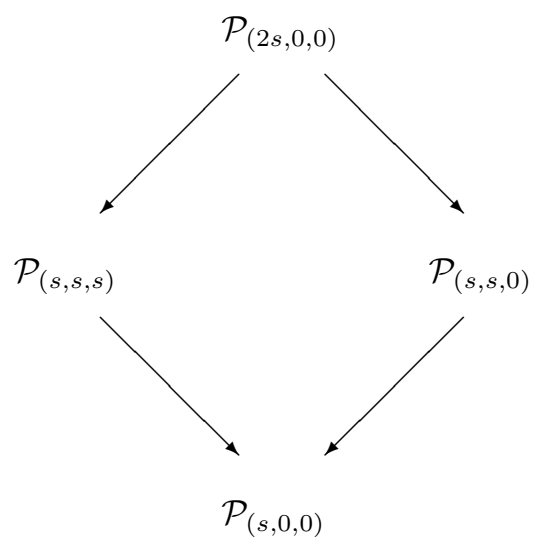

Now let $\mathcal{K}:=\{3,4\}$ be the octahedron with vertices $1, \ldots, 6$, where antipodal vertices are labelled $i, i+3(\bmod 6)$. Then

$$
A(\mathcal{K})=\left\langle\tau_{0}, \tau_{1}, \tau_{2}\right\rangle
$$

with

$$
\begin{gathered}
\tau_{0}:=\left(\begin{array}{ll}
1 & 2
\end{array}\right)\left(\begin{array}{ll}
4 & 5
\end{array}\right), \\
\tau_{1}=\left(\begin{array}{ll}
2 & 3
\end{array}\right)\left(\begin{array}{ll}
5 & 6
\end{array}\right), \\
\tau_{2}=\left(\begin{array}{ll}
3 & 6
\end{array}\right) .
\end{gathered}
$$


The construction of Corollary 5.8 amounts to applying to the Coxeter group $W_{s}=$ $\left\langle\sigma_{0}, \ldots, \sigma_{6}\right\rangle$, with diagram

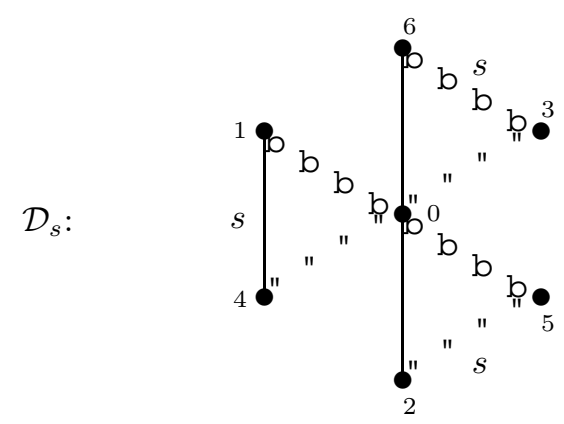

the twisting operation

$$
\left(\sigma_{0}, \ldots, \sigma_{6} ; \tau_{0}, \tau_{1}, \tau_{2}\right) \mapsto\left(\sigma_{0}, \sigma_{1}, \tau_{0}, \tau_{1}, \tau_{2}\right)=:\left(\rho_{0}, \ldots, \rho_{4}\right) .
$$

(The generator $\sigma_{j}$ corresponds to the node marked $j$.) The resulting polytope is $\mathcal{P}_{(2 s, 0,0)}$, with group $A\left(\mathcal{P}_{(2 s, 0,0)}\right)=\left\langle\rho_{0}, \ldots, \rho_{4}\right\rangle=W_{s} \ltimes[3,4]$.

We use the quotient relations (17) to relate the structure of the polytopes $\mathcal{P}_{(s, 0,0)}$, $\mathcal{P}_{(s, s, 0)}$ and $\mathcal{P}_{(s, s, s)}$ to $\mathcal{P}_{(2 s, 0,0)}$. As we shall see, this works particularly well if $s$ is odd; the geometry of the octahedron then comes into play. However, we begin with the case where $s=2 t$ is even, when we shall recognize the group as a certain semi-direct product.

Let us take the generators of $A\left(\mathcal{P}_{(2 s, 0,0)}\right)=W_{2 t} \ltimes[3,4]$ as in (20). If we write $\chi:=(123456)$ and $\omega:=(1245)$, then we have $\rho_{1} \rho_{2} \rho_{3} \rho_{4}=\sigma_{1} \chi$ and $\rho_{1} \rho_{2} \rho_{3} \rho_{4} \rho_{3}=$ $\sigma_{1} \omega$; these elements have orders $12 t$ and $8 t$ in $A\left(\mathcal{P}_{(2 s, 0,0)}\right)$, respectively. By (5), the groups of $\mathcal{P}_{(s, s, s)}$ and $\mathcal{P}_{(s, s, 0)}$ are the Coxeter group [3,4,3,4], factored out by the single extra relations $\left(\rho_{1} \rho_{2} \rho_{3} \rho_{4}\right)^{6 t}=\varepsilon$ and $\left(\rho_{1} \rho_{2} \rho_{3} \rho_{4} \rho_{3}\right)^{4 t}=\varepsilon$, respectively.

But in $A\left(\mathcal{P}_{(2 s, 0,0)}\right)$, we have

$$
\left(\rho_{1} \rho_{2} \rho_{3} \rho_{4}\right)^{6 t}=\left(\sigma_{1} \sigma_{4}\right)^{t}\left(\sigma_{2} \sigma_{5}\right)^{t}\left(\sigma_{3} \sigma_{6}\right)^{t}
$$

and

$$
\left(\rho_{1} \rho_{2} \rho_{3} \rho_{4} \rho_{3}\right)^{4 t}=\left(\sigma_{1} \sigma_{4}\right)^{t}\left(\sigma_{2} \sigma_{5}\right)^{t} .
$$

Therefore, in $A\left(\mathcal{P}_{(s, s, s)}\right)$ and $A\left(\mathcal{P}_{(s, s, 0)}\right)$, these relations impose extra relations on the generators of $W_{2 t}$, namely

$$
\left(\sigma_{1} \sigma_{4}\right)^{t}\left(\sigma_{2} \sigma_{5}\right)^{t}\left(\sigma_{3} \sigma_{6}\right)^{t}=\varepsilon
$$

and

$$
\left(\sigma_{1} \sigma_{4}\right)^{t}\left(\sigma_{2} \sigma_{5}\right)^{t}=\left(\sigma_{1} \sigma_{4}\right)^{t}\left(\sigma_{3} \sigma_{6}\right)^{t}=\left(\sigma_{2} \sigma_{5}\right)^{t}\left(\sigma_{3} \sigma_{6}\right)^{t}=\varepsilon,
$$

respectively; because of the action of $A(\mathcal{K})$, the two latter relations of $(22)$ are equivalent to the first. 
Let $\widehat{W}_{2 t}$ and $\widehat{\widehat{W}}_{2 t}$ denote the quotients of $W_{2 t}$ defined by the extra relations (21) and (22), respectively. Then we have

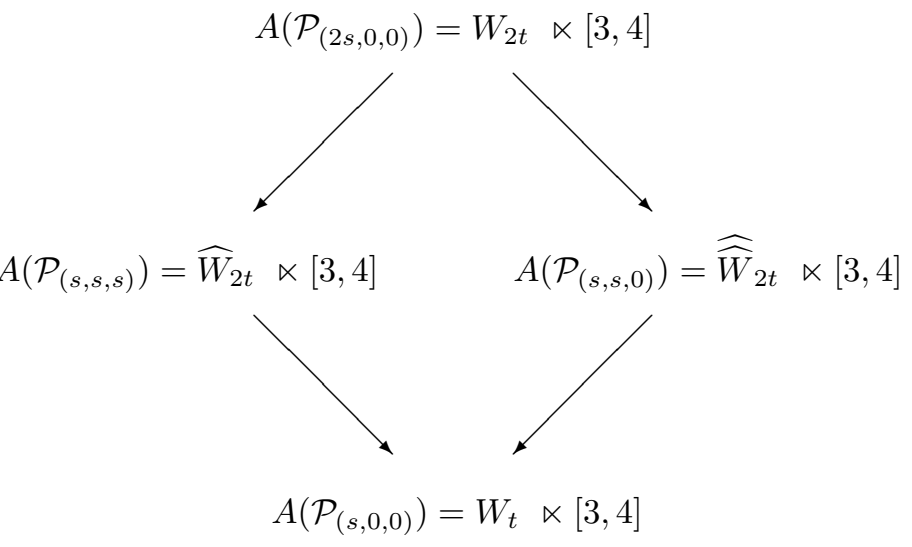

with $s=2 t, t \geq 2$. Note that we indeed have semi-direct products here, because the surjective homomorphisms onto $A\left(\mathcal{P}_{(s, 0,0)}\right)=W_{t} \ltimes[3,4]$ are one-to-one on the $[3,4]$-subgroup.

The more interesting case is when $s=2 t+1(\geq 3)$ is odd. First, we describe a new construction of the polytopes $\mathcal{P}_{(s, 0,0)}$. We begin with two lemmas.

Lemma 6.1. Let $s=2 t+1 \geq 3$, and let $\left\langle\varphi_{0}, \varphi_{1}, \varphi_{2}\right\rangle$ be the Coxeter group with diagram

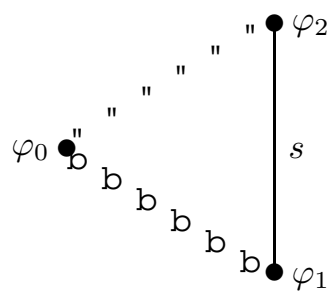

factored out by the extra relation

$$
\left(\varphi_{0} \varphi_{1}\left(\varphi_{2} \varphi_{1}\right)^{t}\right)^{2}=\varepsilon
$$

Then $\left\langle\varphi_{0}, \varphi_{1}, \varphi_{2}\right\rangle \simeq[3, s]$, with the $\psi_{i}$ in the diagram

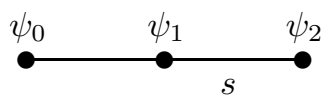

for $[3, s]$ related to the $\varphi_{j}$ by

$$
\begin{aligned}
& \left(\psi_{0}, \psi_{1}, \psi_{2}\right)=\left(\varphi_{0}, \varphi_{1}, \varphi_{1}\left(\varphi_{2} \varphi_{1}\right)^{t}\right), \\
& \left(\varphi_{0}, \varphi_{1}, \varphi_{2}\right)=\left(\psi_{0}, \psi_{1}, \psi_{2} \psi_{1} \psi_{2}\right) .
\end{aligned}
$$

Proof. It is easy to check that the relations of (24) and (25) in terms of $\varphi_{0}, \varphi_{1}$ and $\varphi_{2}$ are equivalent to those for $[3, s]$ in terms of $\psi_{0}, \psi_{1}$ and $\psi_{2}$. 
Lemma 6.2. Let $s=2 t+1 \geq 3$, and let $U_{s}:=\left\langle\varphi_{0}, \varphi_{1}, \ldots, \varphi_{6}\right\rangle$ be the group abstractly defined by the standard relations given by the diagram

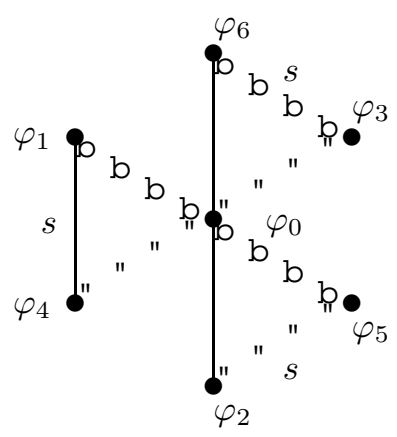

and the three extra relations

$$
\left(\varphi_{0} \varphi_{i}\left(\varphi_{i+3} \varphi_{i}\right)^{t}\right)^{2}=\varepsilon \quad \text { for } i=1,2,3 .
$$

Then $U_{s}$ is isomorphic to the Coxeter group with diagram

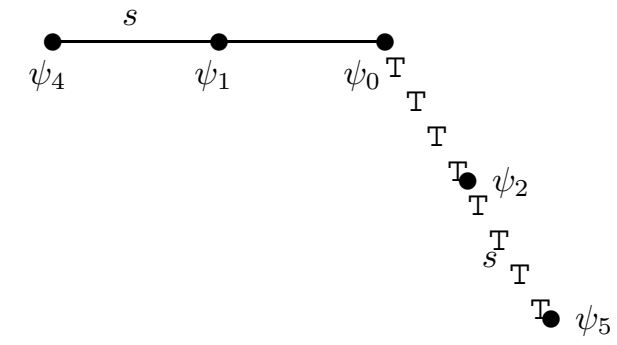

Proof. Start from the group with diagram (28), and apply Lemma 6.1 to each of its subgroups $\left\langle\psi_{0}, \psi_{i}, \psi_{i+3}\right\rangle(i=1,2,3)$. Then the change of generators to $\varphi_{0}, \varphi_{i}$, $\varphi_{i+3}$ in one subgroup does not effect the changes in the other subgroups. It is now straightforward to prove the isomorphism.

The results of the remainder of this section are summarized in Theorem 6.3 below. First, we consider the case $\mathbf{s}=(s, 0,0)$. To construct the universal polytope $\mathcal{P}_{(s, 0,0)}$ with $s=2 t+1$, we begin with the following observation. Relating $\mathcal{P}_{(s, 0,0)}$ to $\mathcal{P}_{(2 s, 0,0)}$ as in $(17)$, we see that in $A\left(\mathcal{P}_{(2 s, 0,0)}\right)=\left\langle\rho_{0}, \ldots, \rho_{4}\right\rangle=W_{s} \ltimes[3,4]$, we have

$$
\left(\rho_{1} \rho_{2} \rho_{3} \rho_{4} \rho_{3} \rho_{2}\right)^{s}=\left(\sigma_{1} \sigma_{4}\right)^{t} \sigma_{1} \cdot \tau_{14}
$$


with $\tau_{14}:=(14)$, so that the imposition of the defining relation $\left(\rho_{1} \rho_{2} \rho_{3} \rho_{4} \rho_{3} \rho_{2}\right)^{s}=\varepsilon$ forces

$$
\left(\sigma_{1} \sigma_{4}\right)^{t} \sigma_{1}=\tau_{14}
$$

in $A\left(\mathcal{P}_{(s, 0,0)}\right)$; here we abuse notation and denote elements in the quotient $A\left(\mathcal{P}_{(s, 0,0)}\right)$ by the same symbols. Then, in view of the action of $A(\mathcal{K})$, we also have

$$
\begin{aligned}
& \left(\sigma_{2} \sigma_{5}\right)^{t} \sigma_{2}=\tau_{25}, \\
& \left(\sigma_{3} \sigma_{6}\right)^{t} \sigma_{3}=\tau_{36}=\tau_{2}=\rho_{4},
\end{aligned}
$$

with $\tau_{25}:=(25)$ and $\tau_{36}:=(36)$. It follows that $\sigma_{0}=\rho_{0}$ and $\left(\sigma_{3} \sigma_{6}\right)^{t} \sigma_{3}=\rho_{4}$ commute, leading to relations like $(25)$. This implies that $W_{s}$ collapses to a quotient of $U_{s}$. As we shall see below, this quotient is indeed $U_{s}$ itself.

We can now construct $\mathcal{P}_{(s, 0,0)}$ using the group $U_{s}=\left\langle\varphi_{0}, \varphi_{1}, \ldots, \varphi_{6}\right\rangle$ in the form (26), and applying an operation like (20), taking into account the identifications that have to occur. More precisely, consider the group $U_{s} \ltimes S_{3}=U_{s} \ltimes\left\langle\tau_{0}, \tau_{1}\right\rangle$ with generators

$$
\left(\alpha_{0}, \ldots, \alpha_{4}\right):=\left(\varphi_{0}, \varphi_{1}, \tau_{0}, \tau_{1},\left(\varphi_{3} \varphi_{6}\right)^{t} \varphi_{3}\right)
$$

Now $A:=\left\langle\alpha_{0}, \ldots, \alpha_{4}\right\rangle$ coincides with $U_{s} \ltimes S_{3}$, since $\tau_{0} \tau_{1} \alpha_{4} \tau_{1} \tau_{0} \varphi_{1}=\left(\varphi_{1} \varphi_{4}\right)^{t} \in A$, and thus $\varphi_{4} \in A$ because $(s, t)=1$ (here, $(s, t)$ is the greatest common divisor). By conjugation, therefore, $\varphi_{1}, \ldots, \varphi_{6} \in A$.

With respect to these generators, $A$ is a string C-group. The facets of the corresponding polytope are polytopes $\{3,4,3\}$, occurring in the form $\{3\}^{\{3\}}$. The vertex-figures are polytopes $\{4,3,4\}_{(s, 0,0)}$, because $\left\langle\alpha_{2}, \alpha_{3}, \alpha_{4}\right\rangle \simeq C_{2}^{3} \ltimes S_{3} \simeq[3,4]$, and $\left\langle\alpha_{1}, \alpha_{2}, \alpha_{3}, \alpha_{4}\right\rangle \simeq\left\langle\varphi_{1}, \ldots, \varphi_{6}\right\rangle \ltimes S_{3}=D_{s}^{3} \ltimes S_{3}$. But our above arguments (preceding this construction) imply that the polytope must in fact be the (universal) $\mathcal{P}_{(s, 0,0)}$ itself, because it covers $\mathcal{P}_{(s, 0,0)}$. In other words, $\mathcal{P}_{(s, 0,0)}$ exists for each odd $s$, and its group is $U_{s} \ltimes S_{3}$. If we set $\widehat{\mathcal{P}}_{(s, 0,0)}:=\mathcal{P}_{(s, 0,0)}$, this proves the case $k=1$ of Theorem 6.3 below.

Next, we treat the case $k=3$ of Theorem 6.3 . We again relate the polytopes $\mathcal{P}_{(s, s, s)}$ with $s=2 t+1$ to $\mathcal{P}_{(2 s, 0,0)}$. In $A\left(\mathcal{P}_{(2 s, 0,0)}\right)=W_{s} \ltimes[3,4]$ we now have

$$
\left(\rho_{1} \rho_{2} \rho_{3} \rho_{4}\right)^{3 s}=\left(\sigma_{1} \sigma_{4}\right)^{t} \sigma_{1} \cdot\left(\sigma_{2} \sigma_{5}\right)^{t} \sigma_{2} \cdot\left(\sigma_{3} \sigma_{6}\right)^{t} \sigma_{3} \cdot \chi^{3},
$$

where $\chi$ is as above, so that $\chi^{3}=(14)(25)(36)$ is the central involution of $[3,4]$. In $A\left(\mathcal{P}_{(2 s, 0,0)}\right)$, the elements $\sigma_{0}=\rho_{0}$ and $\chi^{3}\left(\in\left\langle\rho_{2}, \rho_{3}, \rho_{4}\right\rangle\right)$ commute, so that the imposition of $\left(\rho_{1} \rho_{2} \rho_{3} \rho_{4}\right)^{3 s}=\varepsilon$ leads to

$$
\left(\sigma_{0} \cdot\left(\sigma_{1} \sigma_{4}\right)^{t} \sigma_{1} \cdot\left(\sigma_{2} \sigma_{5}\right)^{t} \sigma_{2} \cdot\left(\sigma_{3} \sigma_{6}\right)^{t} \sigma_{3}\right)^{2}=\varepsilon
$$

in $A\left(\mathcal{P}_{(s, s, s)}\right)$. While it seems difficult to get a handle on the quotient of $W_{s}$ defined by (30), we can still collapse this quotient further onto $U_{s}$, corresponding to passing from $\mathcal{P}_{(s, s, s)}$ to $\mathcal{P}_{(s, 0,0)}$. This suggests constructing from $U_{s}$ a polytope $\widehat{\mathcal{P}}_{(s, s, s)}$ in the class $\left\langle\{3,4,3\},\{4,3,4\}_{(s, s, s)}\right\rangle$; however, this will not coincide with the universal $\mathcal{P}_{(s, s, s)}$.

To do so, consider the regular 3 -simplex $\mathcal{T}$ whose vertices lie at the centres of alternate 2 -faces of $\{3,4\}$, with one of its vertices at the centre of that 2 -face with vertices $1,2,3$. Now the group of the polytope $\widehat{\mathcal{P}}_{(s, s, s)}$ will be $U_{s} \ltimes S_{4}$, with $S_{4}$ realized as the subgroup $S(\mathcal{T})$ of $[3,4]$ which preserves $\mathcal{T}$. Note that $S(\mathcal{T}) \simeq$ 
$[3,4] /\left\langle\chi^{3}\right\rangle$, and $S(\mathcal{T})=\left\langle\tau_{0}, \tau_{1}, \omega^{2}\right\rangle$, with $\omega$ as before, so that $\omega^{2}=(14)(25)$. As generators, we take

$$
\left(\beta_{0}, \ldots, \beta_{4}\right):=\left(\varphi_{0}, \varphi_{1}, \tau_{0}, \tau_{1},\left(\varphi_{1} \varphi_{4}\right)^{t} \varphi_{1} \cdot\left(\varphi_{2} \varphi_{5}\right)^{t} \varphi_{2} \cdot\left(\varphi_{3} \varphi_{6}\right)^{t} \varphi_{3} \cdot \omega^{2}\right) .
$$

In this context, one should think of $\beta_{4}$ as $\chi^{3} \omega^{2}=\tau_{2}$. First note that $A=$ $\left\langle\beta_{0}, \ldots, \beta_{4}\right\rangle$ coincides with $U_{s} \ltimes S(\mathcal{T})$. In fact, $\varphi_{1}, \varphi_{2}, \varphi_{3} \in A$ and $\varphi_{6}=$ $\left(\varphi_{1} \varphi_{2} \varphi_{3} \beta_{4}\right)^{2} \varphi_{3} \in A$; hence, $\varphi_{4}, \varphi_{5} \in A$ and thus $A=U_{s} \ltimes S_{4}$. Again, $A$ is a string C-group, and the facets of the corresponding polytope $\widehat{\mathcal{P}}_{(s, s, s)}$ are isomorphic to $\{3,4,3\}$. Now the group of the vertex-figure is

$$
\left\langle\beta_{1}, \ldots, \beta_{4}\right\rangle=\left\langle\varphi_{1}, \ldots, \varphi_{6}\right\rangle \ltimes S(\mathcal{T}) \simeq D_{s}^{3} \ltimes S_{4} \simeq[4,3,4]_{(s, s, s)},
$$

so that vertex-figures of $\widehat{\mathcal{P}}_{(s, s, s)}$ are isomorphic to $\{4,3,4\}_{(s, s, s)}$. In particular, $\widehat{\mathcal{P}}_{(s, s, s)}$ is an infinite regular polytope in $\left\langle\{3,4,3\},\{4,3,4\}_{(s, s, s)}\right\rangle$ whose group is the semidirect product $U_{s} \ltimes S_{4}$. This proves the case $k=3$ of Theorem 6.3.

In a similar fashion we shall now deal with the case $k=2$, and construct a regular polytope $\widehat{\mathcal{P}}_{(s, s, 0)}$ in $\left\langle\{3,4,3\},\{4,3,4\}_{(s, s, 0)}\right\rangle$, with group $U_{s} \ltimes D_{6}$ (where $s=2 t+1$ is odd $)$. In $A\left(\mathcal{P}_{(2 s, 0,0)}\right)=W_{s} \ltimes[3,4]$, we have

$$
\left(\rho_{1} \rho_{2} \rho_{3} \rho_{4} \rho_{3}\right)^{2 s}=\left(\sigma_{1} \sigma_{4}\right)^{t} \sigma_{1} \cdot\left(\sigma_{2} \sigma_{5}\right)^{t} \sigma_{2} \cdot \widehat{\kappa}_{3},
$$

with $\widehat{\kappa}_{3}:=\omega^{2}=(14)(25)$. In $A\left(\mathcal{P}_{(2 s, 0,0)}\right)$, the elements $\sigma_{0}=\rho_{0}$ and $\omega^{2}$ commute, so that the imposition of $\left(\rho_{1} \rho_{2} \rho_{3} \rho_{4} \rho_{3}\right)^{2 s}=\varepsilon$ leads to

$$
\left(\sigma_{0} \cdot\left(\sigma_{1} \sigma_{4}\right)^{t} \sigma_{1} \cdot\left(\sigma_{2} \sigma_{5}\right)^{t} \sigma_{2}\right)^{2}=\varepsilon
$$

(or equivalent relations modulo [3,4] in $A\left(\mathcal{P}_{(s, 0,0)}\right)$ ). Let $\widehat{\kappa}_{1}:=(25)(36)$ and $\widehat{\kappa}_{2}:=(14)(36) ;$ then $\left\langle\widehat{\kappa}_{1}, \widehat{\kappa}_{2}, \widehat{\kappa}_{3}\right\rangle \simeq C_{2}^{2}$. But in $A\left(\mathcal{P}_{(s, s, 0)}\right)$ we have $\widehat{\kappa}_{3}=\left(\sigma_{1} \sigma_{4}\right)^{t} \sigma_{1}$. $\left(\sigma_{2} \sigma_{5}\right)^{t} \sigma_{2}$ and, by conjugation, $\widehat{\kappa}_{1}=\left(\sigma_{2} \sigma_{5}\right)^{t} \sigma_{2} \cdot\left(\sigma_{3} \sigma_{6}\right)^{t} \sigma_{3}$ and $\widehat{\kappa}_{2}=\left(\sigma_{1} \sigma_{4}\right)^{t} \sigma_{1}$. $\left(\sigma_{3} \sigma_{6}\right)^{t} \sigma_{3}$, so that $\left\langle\widehat{\kappa}_{1}, \widehat{\kappa}_{2}, \widehat{\kappa}_{3}\right\rangle$ becomes a subgroup of the corresponding quotient of $W_{s}$. Finally, for our construction of $\widehat{\mathcal{P}}_{(s, s, 0)}$, we note that in [3,4] the groups $\left\langle\widehat{\kappa}_{1}, \widehat{\kappa}_{2}, \widehat{\kappa}_{3}\right\rangle$ and $\left\langle\tau_{0} \tau_{2}, \tau_{1}\right\rangle=\langle(12)(45)(36),(23)(56)\rangle\left(\simeq D_{6}\right)$ are complementary, with the latter occurring as the group of the Petrie polygon $\mathcal{L}$ of $\{3,4\}$ with successive vertices 1, 2,6, 4, 5,3 (see [5] for the definition, and Figure 1). Again, since it is difficult to identify the quotient of $W_{s}$ defined by (32) and equivalent relations, we collapse the group further onto $U_{s}$, corresponding to passing from $\mathcal{P}_{(s, s, 0)}$ to $\mathcal{P}_{(s, 0,0)}$.

As a subgroup of $[3,4]$, the symmetry group of $\mathcal{L}$ is given by $S(\mathcal{L})=\left\langle\tau_{0} \tau_{2}, \tau_{1}\right\rangle$. Define the elements $\kappa_{1}, \kappa_{2}, \kappa_{3}$ of $U_{s}=\left\langle\varphi_{0}, \ldots, \varphi_{6}\right\rangle$ by

$$
\begin{aligned}
& \kappa_{1}:=\left(\varphi_{2} \varphi_{5}\right)^{t} \varphi_{2} \cdot\left(\varphi_{3} \varphi_{6}\right)^{t} \varphi_{3}, \\
& \kappa_{2}:=\left(\varphi_{1} \varphi_{4}\right)^{t} \varphi_{1} \cdot\left(\varphi_{3} \varphi_{6}\right)^{t} \varphi_{3}, \\
& \kappa_{3}:=\left(\varphi_{1} \varphi_{4}\right)^{t} \varphi_{1} \cdot\left(\varphi_{2} \varphi_{5}\right)^{t} \varphi_{2} .
\end{aligned}
$$

Then $\left\langle\kappa_{1}, \kappa_{2}, \kappa_{3}\right\rangle \simeq C_{2}^{2}$, and $\left\langle\kappa_{1}, \kappa_{2}, \kappa_{3}, \tau_{0} \tau_{2}, \tau_{1}\right\rangle \simeq C_{2}^{2} \ltimes D_{6} \simeq[3,4]$. Now the group of $\widehat{\mathcal{P}}_{(s, s, 0)}$ will be $U_{s} \ltimes D_{6}$, with $D_{6}$ realized as $S(\mathcal{L})$. As generators, we take

$$
\left(\gamma_{0}, \ldots, \gamma_{4}\right):=\left(\varphi_{0}, \varphi_{1}, \tau_{0} \tau_{2} \chi^{3} \kappa_{3}, \tau_{1}, \chi^{3} \kappa_{3}\right)
$$

with $\chi^{3}=(14)(25)(36)$, again as before. Here, $\chi^{3}=\left(\tau_{0} \tau_{2} \tau_{1}\right)^{3} \in S(\mathcal{L})$ and $\tau_{0} \tau_{2} \chi^{3}=(15)(24) \in S(\mathcal{L})$. Keeping in mind the above identifications, one should think of $\gamma_{2}$ as $\tau_{0}\left(=(15)(24) \cdot(14)(25)=\tau_{0} \tau_{2} \chi^{3} \cdot \kappa_{3}\right)$ and of $\gamma_{4}$ as $\tau_{2}(=$ $\left.(14)(25)(36) \cdot(14)(25)=\chi^{3} \cdot \kappa_{3}\right)$. To see that $A:=\left\langle\gamma_{0}, \ldots, \gamma_{4}\right\rangle$ coincides with $U_{s} \ltimes S(\mathcal{L})$, note that $\tau_{0} \tau_{2}=\gamma_{2} \gamma_{4} \in A$, and thus $S(\mathcal{L}) \leq A$; then the conjugates 


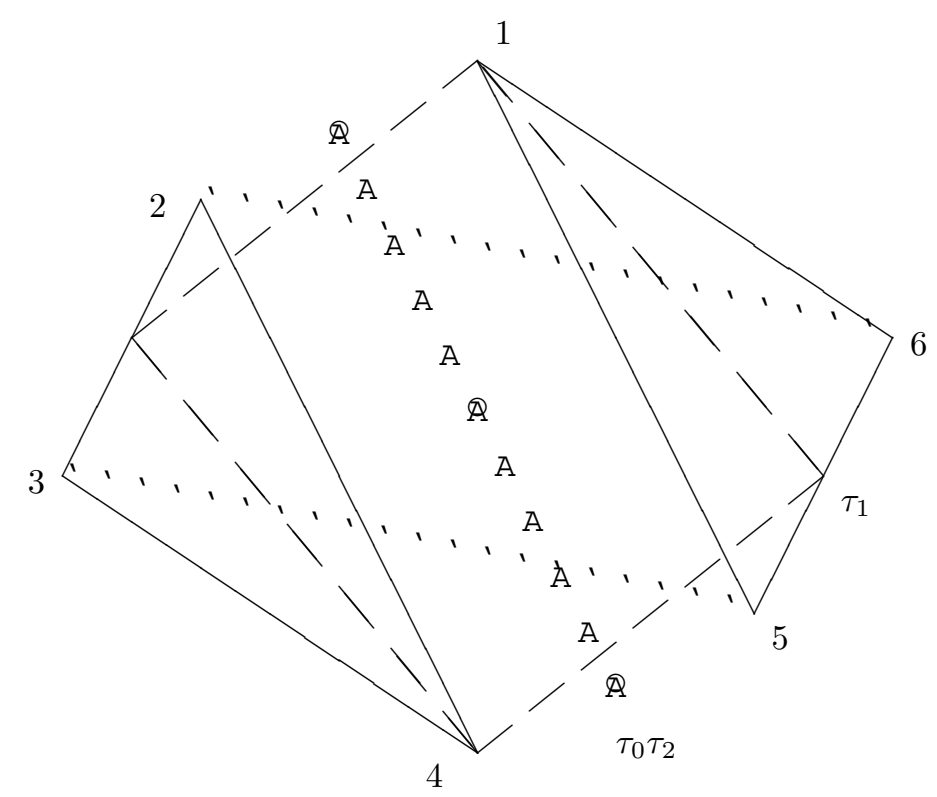

Figure 1. The Petrie polygon $\mathcal{L}$ of $\{3,4\}$.

of $\varphi_{1}$ by elements in $S(\mathcal{L})$ are also in $A$, proving that $A=U_{s} \ltimes S(\mathcal{L})$. Again, $A$ is a string C-group with respect to its generators $\gamma_{0}, \ldots, \gamma_{4}$. To check that the facets of the corresponding polytope $\widehat{\mathcal{P}}_{(s, s, 0)}$ are isomorphic to $\{3,4,3\}$, we note that $\left\langle\gamma_{0}, \ldots, \gamma_{3}\right\rangle=\left\langle\varphi_{0}, \ldots, \varphi_{3}\right\rangle \ltimes S_{3}$, with $S_{3}$ given by $\left\langle\gamma_{2}, \gamma_{3}\right\rangle$. By construction, $\left\langle\gamma_{2}, \gamma_{3}, \gamma_{4}\right\rangle \simeq\left\langle\kappa_{1}, \kappa_{2}, \kappa_{3}\right\rangle \ltimes S(\mathcal{L}) \simeq C_{2}^{2} \ltimes D_{6}=[3,4]$. The vertex-figures are now isomorphic to $\{4,3,4\}_{(s, s, 0)}$, because

$$
\left\langle\gamma_{1}, \ldots, \gamma_{4}\right\rangle=\left\langle\varphi_{1}, \ldots, \varphi_{6}\right\rangle \ltimes S(\mathcal{L})=D_{s}^{3} \ltimes D_{6}=[4,3,4]_{(s, s, 0)} .
$$

It follows that $\widehat{\mathcal{P}}_{(s, s, 0)}$ is in $\left\langle\{3,4,3\},\{4,3,4\}_{(s, s, 0)}\right\rangle$. This completes the proof of the following result.

Theorem 6.3. Let $k=1,2$ or 3 , let $s=2 t+1 \geq 3$, and let $U_{s}$ denote the Coxeter group with diagram (28). Then there exists an infinite regular 5 -polytope $\widehat{\mathcal{P}}_{\left(s^{k}, 0^{3-k}\right)}$ in $\left\langle\{3,4,3\},\{4,3,4\}_{\left(s^{k}, 0^{3-k}\right)}\right\rangle$; its group is $U_{s} \ltimes S_{3}($ for $k=1), U_{s} \ltimes D_{6}$ (for $k=2)$, or $U_{s} \ltimes S_{4}($ for $k=3)$. In particular, $\widehat{\mathcal{P}}_{(s, 0,0)}=\mathcal{P}_{(s, 0,0)}$, the universal member in its class.

Remarks. (a) In the proof of Theorem 6.3 , we identified the groups $[4,3,4]_{\left(s^{k}, 0^{3-k}\right)}$ for odd $s$ as $D_{s}^{3} \ltimes S_{3}$ if $k=1, D_{s}^{3} \ltimes D_{6}$ if $k=2$, or $D_{s}^{3} \ltimes S_{4}$ if $k=3$.

(b) We can also construct a regular 5 -polytope $\widehat{\mathcal{P}}_{(2 s, 0,0)}$ in the class $\langle\{3,4,3\}$, $\left.\{4,3,4\}_{(2 s, 0,0)}\right\rangle$ with group $U_{s} \ltimes[3,4]$, by applying the exact analogue of $(20)$ directly to $U_{s}$. Then (17) remains true with $\mathcal{P}_{\left(s^{k}, 0^{3-k}\right)}$ replaced by $\widehat{\mathcal{P}}_{\left(s^{k}, 0^{3-k}\right)}$, and the corresponding quotient constructions are equivalent to our constructions.

Concluding this section, we remark that our techniques already work in rank 4. For example, if $\mathcal{F}=\{4\}$ and $\mathcal{H}_{s}(\mathcal{F})$ is the diagram on the vertices of a square 
connecting antipodes by a branch labelled $s(\geq 2)$, then by Theorem 5.7

$$
\{3\}^{\mathcal{F}, \mathcal{H}_{s}(\mathcal{F})}=\left\{\{3,4\},\{4,4\}_{(2 s, 0)}\right\}
$$

for all $s$. This amounts to an operation like (20) on a diagram like (19) with only two triangular subdiagrams. For odd $s$, we have an analogue of Lemma 6.2 involving only two tails of (28). The construction of the corresponding polytope is equivalent to that of $[19]$ for $\left\{\{3,4\},\{4,4\}_{(s, 0)}\right\}$.

\section{Polytopes with Small faces}

We often run into the problem of having to exclude the small polytopes of a certain class from our discussion. Examples are the regular polytopes $\left\{\{4,3,4\}_{(2,2,0)}\right.$, $\{3,4,3\}\}$ and $\left\{\{4,3,4\}_{(2,2,2)},\{3,4,3\}\right\}$ and their duals, but also sequences of polytopes of rank 6 . In some cases, the Coxeter-Todd coset enumeration algorithm can be applied to find the order of the corresponding group; even so, the structure of the group has to be determined by other means.

In this section, we identify the groups of several locally toroidal regular polytopes, including these two examples. In particular, this complements the work of [21], which aims at the classification of all such polytopes of rank at least 5 . The basic technique is to construct a polytope as a quotient $2^{\mathcal{K}, \mathcal{D}} / N$ of a larger polytope $2^{\mathcal{K}, \mathcal{D}}$, whose structure we know by Theorem 5.1 and its corollaries. Then the geometry of the vertex-figure is used to identify the new group explicitly. Our approach avoids prior knowledge of the order of the group; thus we need not appeal to the CoxeterTodd algorithm, although it can always be used to check our results. For a general discussion of quotients of polytopes, see [22].

We first describe the basic technique. Let $\mathcal{K}$ be a regular $n$-polytope whose facets $\mathcal{F}$ are centrally symmetric; this assumption on the structure of the facets is important. As before, we denote by $\mathcal{H}_{s}(\mathcal{F})$ the diagram on the vertex set of $\mathcal{F}$ which connects antipodal vertices of $\mathcal{F}$ by a branch labelled $s$, and by $\mathcal{D}_{s}(\mathcal{F})$ the universal $\mathcal{K}$-extension of $\mathcal{H}_{s}(\mathcal{F})$ to the vertex set $V(\mathcal{K})$ of $\mathcal{K}$. We are particularly interested in the case $s=2$, where $\mathcal{H}_{2}(\mathcal{F})$ is the trivial diagram, and thus every restriction of $\mathcal{D}_{2}(\mathcal{F})$ to the vertex-set of a facet of $\mathcal{K}$ is also trivial. We can further note that, by the central symmetry of $\mathcal{F}$, the polytope $2^{\mathcal{F}}$ has a non-standard representation as $2^{\mathcal{F}, \mathcal{H}_{2}(\mathcal{F})}$, which we shall use in our construction ([16, Theorem 3$\left.]\right)$. Then by Theorem 5.1, $\left\{2^{\mathcal{F}}, \mathcal{K}\right\}=\left\{2^{\mathcal{F}, \mathcal{H}_{2}(\mathcal{F})}, \mathcal{K}\right\}=2^{\mathcal{K}, \mathcal{D}_{2}(\mathcal{K})}$. In the above examples, we shall work with $\mathcal{K}=\{3,4,3\}, \mathcal{F}=\{3,4\}$ and $\left\{2^{\mathcal{F}}, \mathcal{K}\right\}=\left\{\{4,3,4\}_{(4,0,0)},\{3,4,3\}\right\}$.

For the remainder of this section we define $\mathcal{H}:=\mathcal{H}_{2}(\mathcal{F})$ and $\mathcal{D}:=\mathcal{D}_{2}(\mathcal{K})$; further, we retain the notation of Section 5. In particular, we have $A(\mathcal{K})=\left\langle\tau_{0}, \ldots, \tau_{n-1}\right\rangle$, and $W(\mathcal{D})=\left\langle\sigma_{i} \mid i \in V(\mathcal{K})\right\rangle$, with $i=0\left(=F_{0}\right)$ the base vertex of $\mathcal{K}$. Then

$$
A\left(2^{\mathcal{K}, \mathcal{D}}\right)=\left\langle\rho_{0}, \ldots, \rho_{n}\right\rangle=W(\mathcal{D}) \ltimes A(\mathcal{K}) .
$$

We shall now construct quotients $2^{\mathcal{K}, \mathcal{D}} / N$ of $2^{\mathcal{K}, \mathcal{D}}$, where $N$ is a subgroup of $W(\mathcal{D})$ which is normal in $A\left(2^{\mathcal{K}, \mathcal{D}}\right)$. We choose $N$ in such a way that the facets $2^{\mathcal{F}}$ of $2^{\mathcal{K}, \mathcal{D}}$ collapse to smaller facets (like $\{4,3,4\}_{(2,2,0)}$ or $\{4,3,4\}_{(2,2,2)}$ in our examples).

Many of these quotients, but not all, are actually also quotients of the polytopes $2^{\mathcal{K}}$. If $\widehat{\mathcal{D}}$ is the trivial diagram on $\mathcal{K}$, and $W(\widehat{\mathcal{D}})=\left\langle\widehat{\sigma}_{i} \mid i \in V(\mathcal{K})\right\rangle$ is the corresponding (abelian) Coxeter group, then $W(\widehat{\mathcal{D}})=C_{2}^{v}$ with $v=|V(\mathcal{K})|$, and $\sigma_{i} \rightarrow \widehat{\sigma}_{i}(i \in V(\mathcal{K}))$ defines a surjective homomorphism $f: W(\mathcal{D}) \rightarrow W(\widehat{\mathcal{D}})$ mapping $N$ onto a subgroup $\widehat{N}$ of $W(\widehat{\mathcal{D}})$ which is normal in $A\left(2^{\mathcal{K}}\right)$. This gives us the 
quotient $2^{\mathcal{K}} / \widehat{N}$. More informally, $\widehat{N}$ will be defined with respect to $W(\widehat{\mathcal{D}})$ in the same way as is $N$ with respect to $W(\mathcal{D})$. Note that if $C_{2}^{v}$ is identified with the $v$-dimensional vector space $G F(2)^{v}$ over the Galois field $G F(2)$, then $\widehat{N}$ is a linear code; see also [27].

The following considerations indicate how the subgroup $N$ of $W(\mathcal{D})$ should be defined in order to induce the right collapse on the facets. Assume that the vertexset of $\mathcal{F}$ is $V(\mathcal{F})=\{0, \ldots, 2 m-1\}$, with $j, j+m$ antipodal vertices of $\mathcal{F}$ for each $j=0, \ldots, m-1$. If $\mathcal{F}$ is the $(n-1)$-crosspolytope $\left\{3^{n-3}, 4\right\}$, then $m=n-1$. Now recall from [16, p.222] that the polytope $2^{\mathcal{F}}=2^{\mathcal{F}, \mathcal{H}}$ admits a realization in euclidean $2 m$-space $E^{2 m}$. In particular, if $e_{0}, \ldots, e_{2 m-1}$ denotes an orthonormal basis of $E^{2 m}$, then the direct product

$$
W(\mathcal{D})=\bigotimes_{j=0}^{m-1}\left\langle\sigma_{j}, \sigma_{j+m}\right\rangle=\left(C_{2} \times C_{2}\right)^{m}
$$

is realized in such a way that $\left\langle\sigma_{j}, \sigma_{j+m}\right\rangle$ acts on the plane $E_{j}=\left\langle e_{j}, e_{j+m}\right\rangle$ of $E^{2 m}=\bigoplus_{j=0}^{m-1} E_{j}$ by

$$
\left\{\begin{aligned}
\sigma_{j}\left(e_{j}\right) & =e_{j+m} \\
\sigma_{j+m}\left(e_{j}\right) & =-e_{j+m}
\end{aligned}\right.
$$

(Recall that each $\sigma_{i}$ is a linear involution.) Here, $\left\langle\sigma_{j}, \sigma_{j+m}\right\rangle$ occurs as a subgroup of index 2 in the symmetry group of the square $P_{j}$ with vertices $\pm e_{j}, \pm e_{j+m}$. Topologically, one can think of the boundary $\partial P_{j}$ of each $P_{j}$ as a 1-sphere (subdivided by vertices), whose product is an $m$-torus $S^{1} \times \cdots \times S^{1}$. Our identifications will now impinge on this $m$-torus to give a new $m$-torus.

Identification vector $\left(2,0^{n-2}\right)$. First we study the smallest possible quotients $2^{\mathcal{K}, \mathcal{D}} / N$, that is, we take the largest possible choice for $N$. In particular, we shall prove Theorem 7.1 below. In our examples, we shall have $\mathcal{F}=\left\{3^{n-3}, 4\right\}$ (with $n \geq 3)$, giving $2^{\mathcal{F}}=\left\{4,3^{n-3}, 4\right\}_{\left(4,0^{n-2}\right)}$. If $\mathcal{F}=\{4\}$, then in $A\left(2^{\mathcal{F}}\right)=\left\langle\rho_{0}, \rho_{1}, \rho_{2}\right\rangle=$ $\left\langle\sigma_{0}, \tau_{0}, \tau_{1}\right\rangle$, we have $\left(\rho_{0} \rho_{1} \rho_{2} \rho_{1}\right)^{2}=\sigma_{0} \sigma_{2}$; hence to collapse the facets onto $\{4,4\}_{(2,0)}$, we have to choose $N$ in such a way that $\sigma_{0} \sigma_{2} \in N$. A similar remark applies more generally with respect to facets $\left\{4,3^{n-3}, 4\right\}_{\left(2,0^{n-2}\right)}$ with identification vector $\left(2,0^{n-2}\right)$, using $\left(\rho_{0} \rho_{1} \cdots \rho_{n-1} \rho_{n-2} \cdots \rho_{1}\right)^{2}=\sigma_{0} \sigma_{n-1}=\sigma_{0} \sigma_{m}$. We therefore define

(36) $\quad N:=\left\langle\varphi \sigma_{i} \sigma_{j} \varphi^{-1}\right| \varphi \in W(\mathcal{D})$, and $\{i, j\}$ antipodal vertices of a facet of $\left.\mathcal{K}\right\rangle$.

Thus $N$ is the normal closure of $\sigma_{0} \sigma_{m}$ in $A\left(2^{\mathcal{K}, \mathcal{D}}\right)$, and

$$
A\left(2^{\mathcal{K}, \mathcal{D}}\right) / N \simeq W(\mathcal{D}) / N \ltimes A(\mathcal{K}) .
$$

Observe that in $W(\mathcal{D}) / N$ we have $\sigma_{i} N=\sigma_{j} N$ if $i, j$ are antipodal vertices of a facet of $\mathcal{K}$.

To find the structure of $W(\mathcal{D}) / N$, define the graph $\mathcal{G}_{\mathcal{K}}$ with vertex-set $V(\mathcal{K})$ as follows. In $\mathcal{G}_{\mathcal{K}}$, two vertices $i, j$ of $V(\mathcal{K})$ are joined by an edge if and only if they are antipodal vertices of a facet of $\mathcal{K}$. Then

$$
W(\mathcal{D}) / N \simeq C_{2}^{\omega\left(\mathcal{G}_{\mathcal{K}}\right)}
$$

with $\omega\left(\mathcal{G}_{\mathcal{K}}\right)$ the number of connected components of $\mathcal{G}_{\mathcal{K}}$. For $(37)$, note that, by the connectivity properties of $\mathcal{K}$, each connected component of $\mathcal{G}_{\mathcal{K}}$ in fact has a representative vertex in the base facet $\mathcal{F}$ of $\mathcal{K}$. But if $i, j \in V(\mathcal{F})$, then $\sigma_{i}$ and $\sigma_{j}$ commute, so that $W(\mathcal{D}) / N$ is abelian. Note that, if $\mathcal{F}=\left\{3^{n-3}, 4\right\}$ (as in our 
examples), we can even find the representative vertex among the vertices $0, \ldots, n-2$ of the base $(n-2)$-face of $\mathcal{F}$.

Further, $A(\mathcal{K})$ acts transitively on the components of $\mathcal{G}_{\mathcal{K}}$. But if $i, j$ are in the same component of $\mathcal{G}_{\mathcal{K}}$, and if $\tau \in A(\mathcal{K})$ with $\tau(i)=i$, then $i, \tau(j)$ and thus $i, j, \tau(j)$ are in the same component of $\mathcal{G}_{\mathcal{K}}$. If $\mathcal{F}=\left\{3^{n-3}, 4\right\}$, we can apply this with $i, j, \tau(j) \in\{0, \ldots, n-2\}$, so that now either $\omega\left(\mathcal{G}_{\mathcal{K}}\right)=1$ or $\omega\left(\mathcal{G}_{\mathcal{K}}\right)=n-1$.

Theorem 7.1. Let $\mathcal{K}$ be a regular n-polytope with facets $\mathcal{F}=\left\{3^{n-3}, 4\right\} \quad(n \geq$ $3)$, and let $\mathcal{G}_{\mathcal{K}}$ be the graph defined above. Then the regular $(n+1)$-polytope $\left\{\left\{4,3^{n-3}, 4\right\}_{\left(2,0^{n-2}\right)}, \mathcal{K}\right\}$ exists if and only if $\omega\left(\mathcal{G}_{\mathcal{K}}\right)=n-1$. In this case the polytope is flat, has group $C_{2}^{n-1} \ltimes A(\mathcal{K})$, and is finite if and only if $\mathcal{K}$ is finite.

Proof. We have $\omega\left(\mathcal{G}_{\mathcal{K}}\right)=1$ or $n-1$. In either case, $A\left(2^{\mathcal{K}, \mathcal{D}}\right) / N$ is a string C-group. If $\omega\left(\mathcal{G}_{\mathcal{K}}\right)=1$, then $W(\mathcal{D}) / N \simeq C_{2}$ and $A\left(2^{\mathcal{K}, \mathcal{D}}\right) / N$ is the group of the regular $(n+1)$-polytope $\left\{\left\{2,3^{n-3}, 4\right\}, \mathcal{K}\right\}$. However, if $\omega\left(\mathcal{G}_{\mathcal{K}}\right)=n-1$, then the facets are flat tori, and $A\left(2^{\mathcal{K}, \mathcal{D}}\right) / N$ is the group of $\left\{\left\{4,3^{n-3}, 4\right\}_{\left(2,0^{n-2}\right)}, \mathcal{K}\right\}$ (we recall that a polytope is flat if each vertex is incident with each facet). The polytope itself is then flat, because it has flat facets.

Corollary 7.2. The regular 4 -polytope $\left\{\{4,4\}_{(2,0)},\{4,4\}_{(p, q)}\right\}$ exists for all $p, q$ except for $q=0$ with $p$ odd. It is finite and flat, and its group is the semi-direct product $C_{2}^{2} \ltimes[4,4]_{(p, q)}$, of order $16\left(p^{2}+q^{2}\right)$.

Proof. Apply Theorem 7.1 with $n=3, \mathcal{K}=\{4,4\}_{(p, q)}$ and $\mathcal{F}=\{4\}$. In particular, $\omega\left(\mathcal{G}_{\mathcal{K}}\right)=1$ if $p$ is odd and $q=0$, and $\omega\left(\mathcal{G}_{\mathcal{K}}\right)=2$ otherwise.

Corollary 7.3. For $n \geq 3$, the regular $(n+1)$-polytope $\left\{\left\{4,3^{n-3}, 4\right\}_{\left(2,0^{n-2}\right)}\right.$, $\left.\left\{3^{n-3}, 4,3\right\}\right\}$ exists and is flat. Its group is $C_{2}^{n-1} \ltimes\left[3^{n-3}, 4,3\right]$.

Proof. In this case, $\mathcal{K}=\left\{3^{n-3}, 4,3\right\}$ and $\omega\left(\mathcal{G}_{\mathcal{K}}\right)=n-1$.

Corollary 7.4. The regular 5-polytope $\left\{\{4,3,4\}_{(2,0,0)},\{3,4,3\}\right\}$ exists and is finite and flat. Its group is $C_{2}^{3} \ltimes[3,4,3]$, of order 9216 .

Proof. This result restates Corollary 7.3 for $\mathcal{K}=\{3,4,3\}$.

Note that another way to construct the (dual of the) polytope in Corollary 7.4 is to let $s=1$ in (19) and (20); this implies that $\sigma_{i}=\sigma_{i+3}$ for $i=1,2,3$.

Corollary 7.5. The regular 6-polytope $\left\{\{4,3,3,4\}_{(2,0,0,0)},\{3,3,4,3\}_{\left(t^{k}, 0^{4-k}\right)}\right\}$ exists for all $t \geq 2$ and $k=1,2$, unless $t$ is odd and $k=1$. It is finite and flat, and its group is $C_{2}^{4} \ltimes[3,3,4,3]_{\left(t^{k}, 0^{4-k}\right)}$, of order $18432 k^{2} t^{4}$.

Proof. Now $\mathcal{K}=\{3,3,4,3\}_{\left(t^{k}, 0^{4-k}\right)}$, so that $\omega\left(\mathcal{G}_{\mathcal{K}}\right)=1$ or 4 . If $k=1$ and $t$ is odd, then $|V(\mathcal{K})|=t^{4}$ is odd and $V(\mathcal{K})$ cannot split into 4 components of the same size; hence $\omega\left(\mathcal{G}_{\mathcal{K}}\right)=1$. In the remaining cases, we can prove that $\omega\left(\mathcal{G}_{\mathcal{K}}\right)=4$. In fact, if $t$ is even (and $k=1$ or 2 ), then the covering $\mu: \mathcal{K} \mapsto\{3,3,4,3\}_{\left(2^{k}, 0^{4-k}\right)}=: \mathcal{L}$ induces an incidence preserving mapping of $\mathcal{G}_{\mathcal{K}}$ onto $\mathcal{G}_{\mathcal{L}}$, and thus $\omega\left(\mathcal{G}_{\mathcal{L}}\right) \leq \omega\left(\mathcal{G}_{\mathcal{K}}\right)$. But a direct calculation shows that $\omega\left(\mathcal{G}_{\mathcal{L}}\right)=4$, so that $\omega\left(\mathcal{G}_{\mathcal{K}}\right)=4$ also. If $k=2$ and $t$ is odd, we can use a covering from $\mathcal{K}$ onto the degenerate "polytope" $\mathcal{L}:=$ $\{3,3,4,3\}_{(1,1,0,0)}$ instead. The 4 vertices of $\mathcal{L}$ are the vertices of a 3 -face, and still represent the 4 connected components of $\mathcal{G}_{\mathcal{L}}$, so that $\omega\left(\mathcal{G}_{\mathcal{K}}\right)=4$ again. 
Identification vector $\left(2,2,0^{n-3}\right)$. We next study quotients whose facets are $\left\{4,3^{n-3}, 4\right\}_{\left(2,2,0^{n-3}\right)}$, with identification vector $\left(2,2,0^{n-3}\right)$. This is more complicated than the previous case. As above, if $\mathcal{F}=\{4\}$, then in $A\left(2^{\mathcal{F}}\right)$ we have $\left(\rho_{0} \rho_{1} \rho_{2}\right)^{4}=\sigma_{0} \sigma_{2} \sigma_{1} \sigma_{3}$ (the order of the latter terms is irrelevant); hence we must have $\sigma_{0} \sigma_{1} \sigma_{2} \sigma_{3} \in N$ to obtain $\{4,4\}_{(2,2)}$. A similar remark also applies to the $(n-1)$-crosspolytope $\mathcal{F}=\left\{3^{n-3}, 4\right\}$ and to the facets $\left\{4,3^{n-3}, 4\right\}_{\left(2,2,0^{n-3}\right)}$, using $\left(\rho_{0} \rho_{1} \rho_{2} \cdots \rho_{n-1} \rho_{n-2} \cdots \rho_{2}\right)^{4}=\sigma_{0} \sigma_{n-1} \sigma_{1} \sigma_{n}$. We therefore define

$$
\begin{aligned}
N:=\left\langle\varphi \sigma_{i} \sigma_{j} \sigma_{k} \sigma_{l} \varphi^{-1}\right| & \varphi \in W(\mathcal{D}) \text { and }\{i, j\},\{k, l\} \text { pairs of } \\
& \text { antipodal vertices in a common facet of } \mathcal{K}\rangle .
\end{aligned}
$$

If $\mathcal{F}=\{4\}$, this is the normal closure of $\sigma_{0} \sigma_{1} \sigma_{2} \sigma_{3}$ in $A\left(2^{\mathcal{K}, \mathcal{D}}\right)$, and the vertices $i, j, k, l$ are in fact all the vertices of the corresponding facet of $\mathcal{K}$; this case would also fit into the discussion below of the identification vector $\left(2^{n-1}\right)$. More generally, if $\mathcal{F}=\left\{3^{n-3}, 4\right\}$, the vertices $i, j, k, l$ are the vertices of a diametral square of a facet of $\mathcal{K}$. Then in $W(\mathcal{D}) / N$, the product of any three generators from $\sigma_{i} N, \sigma_{j} N, \sigma_{k} N$, $\sigma_{l} N$ is the fourth. Again, $A\left(2^{\mathcal{K}, \mathcal{D}}\right) / N \simeq W(\mathcal{D}) / N \ltimes A(\mathcal{K})$. We shall now discuss several applications.

Theorem 7.6. The universal regular 4-polytopes $\left\{\{4,4\}_{(2,2)},\{4,4\}_{(p, q)}\right\}$ exist for all $p, q$. The only finite instances occur for $(p, q)=(2,0),(3,0),(2,2)$ and $(3,3)$, with groups $C_{2}^{3} \ltimes[4,4]_{(2,0)}, C_{2}^{5} \ltimes[4,4]_{(3,0)}, C_{2}^{4} \ltimes[4,4]_{(2,2)}$ and $C_{2}^{6} \ltimes[4,4]_{(3,3)}$, of orders 256, 2304, 1024 and 9216 , respectively.

Proof. For the existence statement we refer to [19], though a direct proof is not difficult here; in fact, in most cases we can appeal to Corollary 7.2 and the quotient lemma, Lemma 2.1. By construction, the polytopes $2^{\mathcal{K}, \mathcal{D}} / N$ are indeed isomorphic to the universal polytopes. It is also known from [19] that finiteness can only occur for the four choices of $(p, q)$. We shall now identify the groups in the finite cases.

The case $\mathcal{K}=\{4,4\}_{(p, 0)}$ with $p=2$ or 3 is special, because $\mathcal{D}$ is trivial and $W(\mathcal{D})$ and $W(\mathcal{D}) / N$ are abelian. Now in $W(\mathcal{D}) / N$, the product of three generators on a facet of $\mathcal{K}$ is the fourth. It follows that $W(\mathcal{D}) / N$ is generated by the 3 elements $\sigma_{i} N$ corresponding to 3 vertices on a facet if $p=2$, and by the 5 elements corresponding to one vertex and its neighbours in $\mathcal{K}$ if $p=3$. It is easy to check that fewer generators will not suffice, so that $W(\mathcal{D}) / N \simeq C_{2}^{3}$ or $C_{2}^{5}$, respectively. Note that the case $\{4,4\}_{(2,0)}$ is also covered by Corollary 7.2 .

Now let $\mathcal{K}=\{4,4\}_{(p, p)}$. Recall that a hole of $\mathcal{K}$ is a path along edges of $\mathcal{K}$ which leaves at each vertex exactly 2 faces to the right. Now through each vertex of $\mathcal{K}$ pass exactly two holes, each of length $2 p$. After $p$ steps, these holes meet again in another vertex which, together with the original vertex, dissects them into halves. It is now easy to see that we can generate $W(\mathcal{D}) / N$ by taking such a pair of holes and choosing on each hole all the vertices from one half, including the two vertices where the holes meet. This gives a set of $2 p$ generators. Further, if $\left\{i_{1}, i_{2}\right\}$ and $\left\{i_{3}, i_{4}\right\}$ are edges $p$ steps apart on a hole of $\mathcal{K}$, then $\sigma_{i_{1}} \sigma_{i_{2}} N=\sigma_{i_{3}} \sigma_{i_{4}} N$; that is, the edges on a hole are identified in pairs, as indicated by parallel heavy edges (and the same labels) in Figure 2, which illustrates the case $p=3$.

For $p=3$, we have generators $\sigma_{i} N$ for $i=0,1,4,5,6,7$. We shall prove that $W(\mathcal{D}) / N$ is elementary abelian of order $2^{6}$. Since $A(\mathcal{K})$ acts on $W(\mathcal{D}) / N$, the 


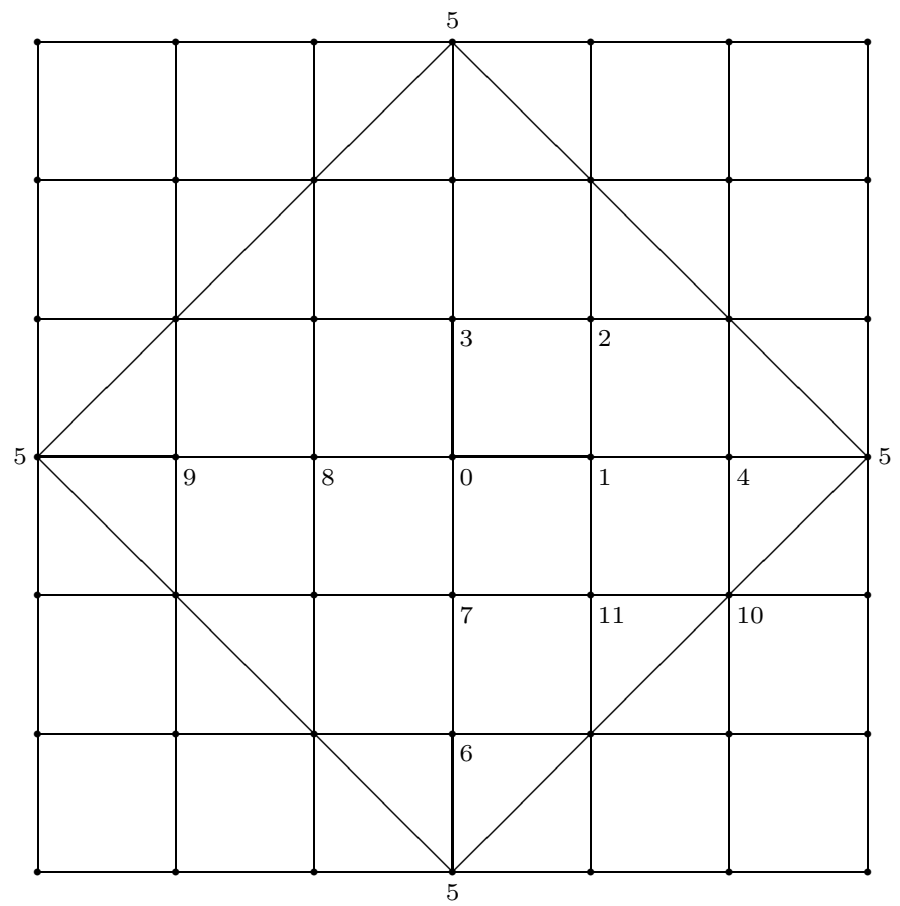

FiguRE 2. Identifications for $p=3$

following imply that $W(\mathcal{D}) / N$ is elementary abelian:

(39)

$$
\begin{gathered}
\sigma_{0} \sigma_{5} N=\sigma_{0} \sigma_{1} N \sigma_{1} \sigma_{4} N \sigma_{4} \sigma_{5} N=\sigma_{5} \sigma_{9} N \sigma_{9} \sigma_{8} N \sigma_{8} \sigma_{0} N=\sigma_{5} \sigma_{0} N \\
\sigma_{0} \sigma_{4} N=\sigma_{0} \sigma_{1} N \sigma_{1} \sigma_{4} N=\sigma_{5} \sigma_{9} N \sigma_{9} \sigma_{8} N=\sigma_{5} \sigma_{8} N=\left(\sigma_{4} \sigma_{8} \sigma_{0}\right) \sigma_{8} N=\sigma_{4} \sigma_{0} N ; \\
\sigma_{0}\left(\sigma_{10} \sigma_{11} \sigma_{1}\right) N=\sigma_{0} \sigma_{4} N=\sigma_{4} \sigma_{0} N=\left(\sigma_{10} \sigma_{11} \sigma_{1}\right) \sigma_{0} N=\sigma_{10} \sigma_{0} \sigma_{11} \sigma_{1} N,
\end{gathered}
$$

so that $\sigma_{0} \sigma_{10} N=\sigma_{10} \sigma_{0} N$, or, equivalently, $\sigma_{4} \sigma_{7} N=\sigma_{7} \sigma_{4} N$. One can also check that no 5 generators suffice, so that indeed $W(\mathcal{D}) / N \simeq C_{2}^{6}$. Here it is helpful to observe that the covering $\mu: \mathcal{K} \mapsto\{4,4\}_{(3,0)}$ induces a surjective homomorphism $\widehat{\mu}: W(\mathcal{D}) / N \mapsto W\left(\mathcal{D}_{(3,0)}\right) / N_{(3,0)} \simeq C_{2}^{5}$, with $\mathcal{D}_{(3,0)}$ and $N_{(3,0)}$ the corresponding diagram and normal subgroup, respectively. But $\operatorname{ker}(\widehat{\mu})$ is non-trivial; in fact, if $i, j$ are 3 steps apart on a hole of $\{4,4\}_{(3,3)}$, then $\widehat{\mu}\left(\sigma_{i} N\right)=\widehat{\mu}\left(\sigma_{j} N\right)$, and thus $\sigma_{i} \sigma_{j} N \in \operatorname{ker}(\widehat{\mu})$. Let $\widetilde{N}$ denote the normal subgroup of (36) defined with respect to $\mathcal{K}$, and let $\mathcal{G}_{\mathcal{K}}$ be the corresponding graph. Now if $\sigma_{i} \sigma_{j} N=N$, then $\sigma_{i} \sigma_{j} \widetilde{N}=\widetilde{N}$, contradicting the fact that $i, j$ lie in different components of $\mathcal{G}_{\mathcal{K}}$. Hence $\sigma_{i} \sigma_{j} N$ is a non-trivial element of $\operatorname{ker}(\widehat{\mu})$, proving that $W(\mathcal{D}) / N \simeq C_{2}^{6}$.

If $p=2$, we have 4 generators, and an analogue of $(39)$ shows that $W(\mathcal{D}) / N$ is elementary abelian. Again one can check that 3 generators do not suffice. This completes the proof.

We next apply the method to polytopes of type $\left\{3^{n-3}, 4,3\right\}$. Here, we begin with the following lemma. 
Lemma 7.7. For $n \geq 3$, let $\mathcal{K}$ be a regular n-polytope of type $\left\{3^{n-3}, 4,3\right\}$ with facets $\mathcal{F}=\left\{3^{n-3}, 4\right\}$, and let $N$ be as in (38). If $\left\{i_{1}, \ldots, i_{n-2}, j\right\}$ with $j=$ $i_{n-1}, i_{n}, i_{n+1}$ are three simplicial $(n-2)$-faces of $\mathcal{K}$ with a common $(n-3)$-face, then $W(\mathcal{D}) / N=\left\langle\sigma_{i_{1}} N, \ldots, \sigma_{i_{n+1}} N\right\rangle$. In particular, $W(\mathcal{D}) / N$ is elementary abelian of order at most $2^{n+1}$.

Proof. First consider the facet $\left\{3^{n-3}, 4\right\}$ of $\mathcal{K}$ which contains the adjacent $(n-2)$ faces $\left\{i_{1}, \ldots, i_{n-2}, i_{n-1}\right\}$ and $\left\{i_{1}, \ldots, i_{n-2}, i_{n}\right\}$. Then the subgroup of $W(\mathcal{D}) / N$ induced by this facet can be generated by $\sigma_{i_{1}} N, \ldots, \sigma_{i_{n}} N$. Indeed, recall that by (38) the product of the generators corresponding to three vertices of a diametral square of a facet is the fourth.

Clearly we should expect at least one more generator to obtain $W(\mathcal{D}) / N$ itself. However, since $\left\{i_{1}, \ldots, i_{n-2}, i_{n+1}\right\}$ is the third $(n-2)$-face of $\mathcal{K}$ containing the $(n-3)$-face $\left\{i_{1}, \ldots, i_{n-2}\right\}$, we now see that $W(\mathcal{D}) / N=\left\langle\sigma_{i_{k}} N \mid k=1, \ldots, n+1\right\rangle$. The reason is straightforward; as above, once we have $n$ generators on a facet corresponding to adjacent $(n-2)$-faces, we have them all, and so we can move from facet to facet of $\mathcal{K}$, starting with the three which contain $\left\{i_{1}, \ldots, i_{n-2}\right\}$. Since in $\mathcal{K}$ there are only three facets surrounding an $(n-3)$-face, this process covers all the facets of $\mathcal{K}$. But now each pair of $\sigma_{i_{1}} N, \ldots, \sigma_{i_{n+1}} N$ belongs to some common facet, and so they commute. It follows that $W(\mathcal{D}) / N$ is elementary abelian, of order at most $2^{n+1}$.

By Lemma 7.7, if $\mathcal{K}$ is a regular $n$-polytope of type $\left\{3^{n-3}, 4,3\right\}$ with crosspolytopes $\left\{3^{n-3}, 4\right\}$ as facets, then $A\left(2^{\mathcal{K}, \mathcal{D}}\right) / N \simeq C_{2}^{m} \ltimes A(\mathcal{K})$ with $m \leq n+1$. Using the natural identification of the automorphism group of $C_{2}^{m}$ with the general linear group $G L_{m}(2)$ over $G F(2)$, this semi-direct product defines a representation

$$
r: A(\mathcal{K}) \rightarrow G L_{m}(2) .
$$

In particular, the generators $\tau_{0}, \ldots, \tau_{n-1}$ of $A(\mathcal{K})$ are represented by $m \times m$ matrices over $G F(2)$. If $m=n+1$, then the generators $\sigma_{i_{1}} N, \ldots, \sigma_{i_{n+1}} N$ of $W(\mathcal{D}) / N$ correspond to a basis of the $(n+1)$-dimensional vector space over $G F(2)$, so that we can express $\tau_{0}, \ldots, \tau_{n-1}$ as matrices with respect to this basis. Below we shall use this approach to prove existence and non-existence of certain polytopes.

Theorem 7.8. For $n \geq 3$, let $\mathcal{K}$ be a regular $n$-polytope of type $\left\{3^{n-3}, 4,3\right\}$ with facets $\mathcal{F}=\left\{3^{n-3}, 4\right\}$, and let $N$ be as in $(38)$. If the regular $(n+1)$-polytope $\left\{\left\{4,3^{n-3}, 4\right\}_{\left(2,0^{n-2}\right)}, \mathcal{K}\right\}$ exists (with group $C_{2}^{n-1} \ltimes A(\mathcal{K})$ ), then $W(\mathcal{D}) / N \simeq C_{2}^{m}$ with $m=n+1$ or $n-1$. In particular, the regular $(n+1)$-polytope $\left\{\left\{4,3^{n-3}, 4\right\}_{\left(2,2,0^{n-3}\right)}, \mathcal{K}\right\}$ also exists if and only if $W(\mathcal{D}) / N \simeq C_{2}^{n+1}$, in which case its group is $C_{2}^{n+1} \ltimes A(\mathcal{K})$.

Proof. Let $\tilde{N}$ be the normal subgroup defined as in (36), and let $\mathcal{G}_{\mathcal{K}}$ be the associated graph. By Theorem 7.1, $\omega\left(\mathcal{G}_{\mathcal{K}}\right)=n-1$ and $W(\mathcal{D}) / \widetilde{N} \simeq C_{2}^{n-1}$. Clearly there is a homomorphism of $A\left(2^{\mathcal{K}, \mathcal{D}}\right) / N=\left\langle\rho_{0}, \ldots, \rho_{n}\right\rangle$ onto the group $A\left(2^{\mathcal{K}, \mathcal{D}}\right) / \widetilde{N}$ of the polytope $\left\{\left\{4,3^{n-3}, 4\right\}_{\left(2,0^{n-2}\right)}, \mathcal{K}\right\}$. By Lemma 2.1, if the subgroup $\left\langle\rho_{0}, \ldots, \rho_{n-1}\right\rangle$ is isomorphic to $\left[4,3^{n-3}, 4\right]_{\left(2,2,0^{n-3}\right)}$, then the polytope $\left\{\left\{4,3^{n-3}, 4\right\}_{\left(2,2,0^{n-3}\right)}, \mathcal{K}\right\}$ exists, and $A\left(2^{\mathcal{K}, \mathcal{D}}\right) / N$ is its group. In this case, $N \neq \tilde{N}$, and the canonical projection $W(\mathcal{D}) / N \mapsto W(\mathcal{D}) / \widetilde{N}$ is not an isomorphism. Conversely, if the polytope $\left\{\left\{4,3^{n-3}, 4\right\}_{\left(2,2,0^{n-3}\right)}, \mathcal{K}\right\}$ exists, then the subgroup $\left\langle\rho_{0}, \ldots, \rho_{n-1}\right\rangle$ of its group $A\left(2^{\mathcal{K}, \mathcal{D}}\right) / N$ is isomorphic to $\left[4,3^{n-3}, 4\right]_{\left(2,2,0^{n-3}\right)}$. 
Let $\left\{i_{1}, \ldots, i_{n-2}\right\}$ be an $(n-3)$-face of $\mathcal{K}$, and let $\left\{i_{1}, \ldots, i_{n-2}, j\right\}$ with $j=$ $i_{n-1}, i_{n}, i_{n+1}$ be the surrounding $(n-2)$-faces. By Lemma 7.7,

$$
W(\mathcal{D}) / N=\left\langle\sigma_{i_{1}} N, \ldots, \sigma_{i_{n+1}} N\right\rangle,
$$

an elementary abelian group of order at most $2^{n+1}$. The arguments above show that its order is at least $2^{n-1}$.

To begin with, we assume that $\left\langle\rho_{0}, \ldots, \rho_{n-1}\right\rangle \simeq\left[4,3^{n-3}, 4\right]_{\left(2,2,0^{n-3}\right)}$. Then the subgroup of $W(\mathcal{D}) / N$ induced by the base facet $\mathcal{F}$ of $\mathcal{K}$ has order $2^{n}$, because it defines the base facet of $\left\{\left\{4,3^{n-3}, 4\right\}_{\left(2,2,0^{n-3}\right)}, \mathcal{K}\right\}$. We shall prove that $W(\mathcal{D}) / N \simeq$ $C_{2}^{n+1}$. First note that the stabilizer in $A(\mathcal{K})$ of the $(n-3)$-face $\left\{i_{1}, \ldots, i_{n-2}\right\}$ acts on the $n+1$ generators $\sigma_{i_{1}} N, \ldots, \sigma_{i_{n+1}} N$ like a group $S_{n-2} \times S_{3}$. If the order is not $2^{n+1}$, then there is a non-trivial relation between the generators, and any such relation involves all the first $n-2$ generators or all the last three. In fact, if $i, j \in\left\{i_{1}, \ldots, i_{n-2}\right\}$ or $i, j \in\left\{i_{n-1}, i_{n}, i_{n+1}\right\}$, and if $\sigma_{i} N$ is involved but $\sigma_{j} N$ is not, then the transposition $(i j)$ in $S_{n-2} \times S_{3}$ maps the given relation onto a new relation in which $i$ is replaced by $j$; taking the product gives the relation $\sigma_{i} \sigma_{j} N=N$, or $\sigma_{i} \sigma_{j} \in N$. But no relation can involve only the first $n$ generators, so that this gives a contradiction if $i, j \in\left\{i_{1}, \ldots, i_{n-2}\right\}$. If $i, j \in\left\{i_{n-1}, i_{n}, i_{n+1}\right\}$, then $\sigma_{i} \sigma_{j}$ generates $\widetilde{N}$ as a normal subgroup, so that $\sigma_{i} \sigma_{j} \in N$ implies $N=\widetilde{N}$, again a contradiction.

This leaves us with two possible relations. We prove that each leads to a contradiction modulo $\tilde{N}$. The first, $\sigma_{i_{n-1}} \sigma_{i_{n}} \sigma_{i_{n+1}} N=N$, cannot occur, because modulo $\widetilde{N}$ the three generators are the same but are not trivial. The second, $\sigma_{i_{1}} \cdots \sigma_{i_{n+1}} N=N$, implies that $\sigma_{i_{1}} \cdots \sigma_{i_{n-1}} \widetilde{N}=\sigma_{i_{1}} \cdots \sigma_{i_{n-1}} \sigma_{i_{n}} \sigma_{i_{n+1}} \widetilde{N}=\widetilde{N}$; again this is a contradiction, because modulo $\widetilde{N}$ there cannot be a relation involving only the first $n-1$ generators. It follows that $W(\mathcal{D}) / N$ must indeed have order $2^{n+1}$.

Finally, if the subgroup $\left\langle\rho_{0}, \ldots, \rho_{n-1}\right\rangle$ is a proper quotient of $\left[4,3^{n-3}, 4\right]_{\left(2,2,0^{n-3}\right)}$, it must be isomorphic to $\left[4,3^{n-3}, 4\right]_{\left(2,0^{n-2}\right)}$. Now the subgroup $\left\langle\sigma_{i_{1}} N, \ldots, \sigma_{i_{n}} N\right\rangle$ representing a facet of $\mathcal{K}$ can already be generated by the generators $\sigma_{i_{1}} N, \ldots$, $\sigma_{i_{n-1}} N$ corresponding to the $(n-2)$-face $\left\{i_{1}, \ldots, i_{n-1}\right\}$ of this facet. It follows that this is so for all subgroups representing facets, and therefore

$$
W(\mathcal{D}) / N=\left\langle\sigma_{i_{1}} N, \ldots, \sigma_{i_{n-1}} N\right\rangle \simeq C_{2}^{n-1}
$$

(and $N=\tilde{N})$. This completes the proof.

Corollary 7.9. For $n \geq 3$, the regular $(n+1)$-polytope $\left\{\left\{4,3^{n-3}, 4\right\}_{\left(2,2,0^{n-3}\right)}\right.$, $\left.\left\{3^{n-3}, 4,3\right\}\right\}$ exists, and has group $C_{2}^{n+1} \ltimes\left[3^{n-3}, 4,3\right]$.

Proof. Apply Theorem 7.8 with $\mathcal{K}=\left\{3^{n-3}, 4,3\right\}$, and use Corollary 7.3. We need to prove that $W(\mathcal{D}) / N \simeq C_{2}^{n+1}$.

Assume for now that $W(\mathcal{D}) / N \simeq C_{2}^{n+1}$, and consider the representation $r$ : $A(\mathcal{K})=\left[3^{n-3}, 4,3\right] \longmapsto G L_{n+1}(2)$. Let $i_{1}, \ldots, i_{n+1}$ be as in Lemma 7.7. Let $F_{n-1}^{1}, F_{n-1}^{2}, F_{n-1}^{3}$ be the facets of $\mathcal{K}$ surrounding the $(n-3)$-face $\left\{i_{1}, \ldots, i_{n-2}\right\}$, such that $\left\{i_{1}, \ldots, i_{n-2}, i_{n-2+j}\right\}$ and $\left\{i_{1}, \ldots, i_{n-2}, i_{n-1+j}\right\}$ are $(n-2)$-faces of $F_{n-1}^{j}$ (with $j+1$ taken modulo 3 ). For $l=1, \ldots, n-2$ and $j=1,2,3$, let $i_{l, j}$ denote the vertex of $F_{n-1}^{j}$ antipodal to $i_{l}$. The generators $\sigma_{i_{1}} N, \ldots, \sigma_{i_{n+1}} N$ of $W(\mathcal{D}) / N$ correspond to a basis of $G F(2)^{n+1}$, and the images under $r$ of the generators $\tau_{0}, \ldots, \tau_{n-1}$ of $A(\mathcal{K})$ are uniquely determined by their effect on this basis. Thus to find the matrices for 
$r\left(\tau_{0}\right), \ldots, r\left(\tau_{n-1}\right)$, it suffices to consider how $\tau_{0}, \ldots, \tau_{n-1}$ act on $\sigma_{i_{1}} N, \ldots, \sigma_{i_{n+1}} N$, with identification modulo $N$ understood.

Using the geometry of $\mathcal{K}$, we can express $\tau_{0}, \ldots, \tau_{n-1}$ as permutations of $i_{1}, \ldots$, $i_{n+1}$ and of some of the $i_{l, j}$, as follows:

$$
\left\{\begin{aligned}
\tau_{p} & =\left(i_{p+1} i_{p+2}\right) \quad \text { for } 0 \leq p \leq n-1, p \neq n-3 \\
\tau_{n-3} & =\left(i_{n-2} i_{n-1}\right)\left(i_{n} i_{n-2,1}\right)\left(i_{n+1} i_{n-2,3}\right)
\end{aligned}\right.
$$

The matrices of $r\left(\tau_{p}\right)$ with $p \neq n-3$ are now just the corresponding $(n+1) \times$ $(n+1)$ permutation matrices. However, for $\tau_{n-3}$ we need the relations $\sigma_{i_{n-2,1}} N=$ $\sigma_{i_{n-2}} \sigma_{i_{n-1}} \sigma_{i_{n}} N$ and $\sigma_{i_{n-2,3}} N=\sigma_{i_{n-2}} \sigma_{i_{n-1}} \sigma_{i_{n+1}} N$, to find the $(n+1) \times(n+1)$ matrix

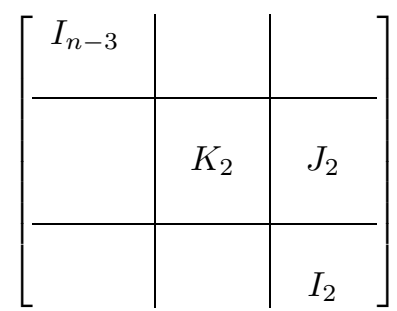

of $r\left(\tau_{n-3}\right)$. Here, only non-zero entries are indicated; in particular, $I_{k}$ is the $k \times k$ identity matrix, and

$$
K_{2}:=\left[\begin{array}{ll}
0 & 1 \\
1 & 0
\end{array}\right], \quad J_{2}:=\left[\begin{array}{ll}
1 & 1 \\
1 & 1
\end{array}\right] .
$$

Hence, if $W(\mathcal{D}) / N \simeq C_{2}^{n+1}$, then the representation $r$ is given by these matrices for $\tau_{0}, \ldots, \tau_{n-1}$.

We can now proceed as follows. Since the above matrices satisfy all the defining relations for the Coxeter group $\left[3^{n-3}, 4,3\right]$, they therefore define a representation $r^{\prime}$ : $A(\mathcal{K}) \rightarrow G L_{n+1}(2)$, and thus a semi-direct product $C_{2}^{n+1} \ltimes A(\mathcal{K})$. This semi-direct product satisfies all the defining relations for the group of $\left\{\left\{4,3^{n-3}, 4\right\}_{\left(2,2,0^{n-3}\right)}, \mathcal{K}\right\}$, and so must be isomorphic to this group. It follows that the polytope exists and has group $C_{2}^{n+1} \ltimes A(\mathcal{K})$, as required; further, $r^{\prime}=r$.

Corollary 7.10. The regular 5-polytope $\left\{\{4,3,4\}_{(2,2,0)},\{3,4,3\}\right\}$ exists and is finite. Its group is $C_{2}^{5} \ltimes[3,4,3]$, of order 36864 .

Proof. This result restates Corollary 7.9 for $\mathcal{K}=\{3,4,3\}$.

Corollary 7.11. The regular 6-polytope $\left\{\{4,3,3,4\}_{(2,2,0,0)},\{3,3,4,3\}_{\left(t^{k}, 0^{4-k}\right)}\right\}$, with $t \geq 2$ and $k=1$ or 2 , exists if and only if $t$ is even. In this case, the polytope is finite, and its group is $C_{2}^{6} \ltimes[3,3,4,3]_{\left(t^{k}, 0^{4-k}\right)}$ of order $73728 k^{2} t^{4}$.

Proof. Now $\mathcal{K}=\{3,3,4,3\}_{\left(t^{k}, 0^{4-k}\right)}$ and $n=5$. If $t$ is even, then the covering $\mu: \mathcal{K} \rightarrow\{3,3,4,3\}_{(2,0,0,0)}$ induces a surjective homomorphism $W(\mathcal{D}) / N \rightarrow$ $W\left(\mathcal{D}^{\prime}\right) / N^{\prime}$, with $\mathcal{D}^{\prime}$ and $N^{\prime}$ the diagram and normal subgroup defined with respect to $\{3,3,4,3\}_{(2,0,0,0)}$. The two polytopes

$$
\left\{\{4,3,3,4\}_{(2,0,0,0)}, \mathcal{K}\right\} \text { and }\left\{\{4,3,3,4\}_{(2,0,0,0)},\{3,3,4,3\}_{(2,0,0,0)}\right\}
$$

exist by Corollary 7.5; thus Theorem 7.8 applies. In particular, if $W\left(\mathcal{D}^{\prime}\right) / N^{\prime} \simeq C_{2}^{6}$, then $W(\mathcal{D}) / N \simeq C_{2}^{6}$, and the polytope $\left\{\{4,3,3,4\}_{(2,2,0,0)}, \mathcal{K}\right\}$ also exists, with group $C_{2}^{6} \ltimes A(\mathcal{K})$. But the case $\{3,3,4,3\}_{(2,0,0,0)}$ can now be handled directly, using the same arguments as for $\{3,3,4,3\}$ in the proof of Corollary 7.9. In particular, 
$W\left(\mathcal{D}^{\prime}\right) / N^{\prime} \simeq C_{2}^{6} ;$ to confirm this, the Coxeter-Todd algorithm predicts $2^{6}$ vertices for the polytope. This settles the case where $t$ is even.

Now let $t$ be odd and $k=2$. Again, the regular polytope $\left\{\{4,3,3,4\}_{(2,0,0,0)}, \mathcal{K}\right\}$ exists by Corollary 7.5, so that Theorem 7.8 implies that $W(\mathcal{D}) / N \simeq C_{2}^{m}$ with $m=4$ or 6 . For the non-existence of $\left\{\{4,3,3,4\}_{(2,2,0,0)}, \mathcal{K}\right\}$, it suffices to prove that $m \neq 6$.

Assume that $m=6(=n+1)$. We can now use the representation $r: A(\mathcal{K}) \mapsto$ $G L_{6}(2)$, as in the proof of Corollary 7.9. In particular, (40) still holds (with $n=5$ ), as does the representation of $r\left(\tau_{0}\right), \ldots, r\left(\tau_{n-1}\right)$ by matrices.

Once the matrices are found, we can proceed as follows. Consider the element $\alpha:=\left(\tau_{0} \cdot \tau_{1} \tau_{2} \tau_{3} \tau_{2} \tau_{1} \cdot \tau_{4} \tau_{3} \tau_{2} \tau_{3} \tau_{4}\right)^{2}$, which is a "translation" of $\mathcal{K}$. By (6), this has order $t$; indeed, since $k=2$, we know that $\alpha^{t}=\varepsilon$ is the only extra defining relation for $A(\mathcal{K})$. It is now straightforward to compute the matrix of $r(\alpha)$ using the matrices for $r\left(\tau_{0}\right), \ldots, r\left(\tau_{n-1}\right)$. However, we find that this matrix has period 2. This is a contradiction, because the period must also divide the period $t$ of $\alpha$. It follows that we cannot have $m=6$. This settles the case where $t$ is odd and $k=2$.

Finally, let $t$ be odd and $k=1$. Now we cannot appeal to Corollary 7.5 and Theorem 7.8. Assume that $\left\{\{4,3,3,4\}_{(2,2,0,0)},\{3,3,4,3\}_{(t, 0,0,0)}\right\}$ exists. Then the subgroup $\left\langle\rho_{0}, \ldots, \rho_{4}\right\rangle$ of its group $A\left(2^{\mathcal{K}, \mathcal{D}}\right) / N$ is isomorphic to $[4,3,3,4]_{(2,2,0,0)}$, so that the subgroup of $W(\mathcal{D}) / N$ induced by a facet of $\mathcal{K}$ has order $2^{5}$. Hence $W(\mathcal{D}) / N \simeq C_{2}^{m}$ with $m=5$ or 6 . Now the assumption $m=6$ can be refuted as above, using the same translation $\alpha$. Note here that $\alpha$ still has order $t$, although $\alpha^{t}=\varepsilon$ is not the extra defining relation if $k=1$.

If $m=5$, we can argue as in the proof of Theorem 7.8 that any non-trivial relation between the generators $\sigma_{i_{1}} N, \ldots, \sigma_{i_{6}} N$ must involve all of the first three generators, or all of the last three. (Note that now $\omega\left(\mathcal{G}_{\mathcal{K}}\right)=1$ and $W(\mathcal{D}) / \widetilde{N} \simeq C_{2}$, and hence $N \neq \tilde{N}$.) But any relation involving only three generators leads to a contradiction, because modulo $\widetilde{N}$ the generators are the same but are not trivial. Hence we are left with $\sigma_{i_{1}} \cdots \sigma_{i_{6}} N=N$, or, equivalently, $\sigma_{i_{6}} N=\sigma_{i_{1}} \cdots \sigma_{i_{5}} N$. In particular, $W(\mathcal{D}) / N=\left\langle\sigma_{i_{1}} N, \ldots, \sigma_{i_{5}} N\right\rangle$.

We can now work with a representation $r: A(\mathcal{K}) \rightarrow G L_{5}(2)$. Using the same notation $F_{4}^{j}\left(=F_{n-1}^{j}\right)$ and $i_{l, j}$ as before, and observing that $r\left(\tau_{p}\right)$ is now uniquely determined by the effect $\tau_{p}$ has on $\sigma_{i_{1}} N, \ldots, \sigma_{i_{5}} N$, we arrive at the following permutations: $\tau_{0}, \tau_{1}, \tau_{3}, \tau_{4}$ as in (40) with $n=5$; and $\tau_{2}=\left(i_{3} i_{4}\right)\left(i_{5} i_{3,1}\right)$. Now $\tau_{0}, \tau_{1}, \tau_{3}$ are represented by the corresponding $5 \times 5$ permutation matrices. For $\tau_{2}$ and $\tau_{4}$, we can use the relations $\sigma_{i_{3,1}} N=\sigma_{i_{3}} \sigma_{i_{4}} \sigma_{i_{5}} N$ and $\sigma_{i_{6}} N=\sigma_{i_{1}} \cdots \sigma_{i_{5}} N$ to find the matrices

$$
\left[\begin{array}{lllll}
1 & 0 & 0 & 0 & 0 \\
0 & 1 & 0 & 0 & 0 \\
0 & 0 & 0 & 1 & 1 \\
0 & 0 & 1 & 0 & 1 \\
0 & 0 & 0 & 0 & 1
\end{array}\right], \quad\left[\begin{array}{lllll}
1 & 0 & 0 & 0 & 1 \\
0 & 1 & 0 & 0 & 1 \\
0 & 0 & 1 & 0 & 1 \\
0 & 0 & 0 & 1 & 1 \\
0 & 0 & 0 & 0 & 1
\end{array}\right]
$$

respectively. If $\alpha$ is the same translation as above, then the matrix of $r(\alpha)$ again has period 2, which gives a contradiction. It follows that we cannot have $m=5$. This completes the proof.

For Corollary 7.11, note that faster non-existence proofs are available if $t$ is an odd prime with $t \neq 3,5,7,31$. Let $\mathcal{K}:=\{3,3,4,3\}_{(t, 0,0,0)}$. By Lemma 7.7, we have 
a representation $r: A(\mathcal{K}) \rightarrow G L_{m}(2)$ with $m \leq 6$. In $\mathcal{K}$, the vertex $i_{1}$ can be mapped to any of its neighbours by a "translation" $\beta$ (say) of $\mathcal{K}$. If $t$ is a prime, then $r(\beta)$ is trivial or $r(\beta)$ has order $t$; in the latter case, $t$ divides the order of $G L_{m}(2)$. But for $2 \leq m \leq 6$, the only odd primes $t$ which can divide the order of $G L_{m}(2)$ are $3,5,7$ or 31 , and these are excluded. On the other hand, $i_{2}, \ldots, i_{6}$ are neighbours of $i_{1}$; hence, if $m \geq 2$ and $\beta$ maps $i_{1}$ to a vertex from $i_{2}, \ldots, i_{6}$, then $r(\beta)$ cannot be trivial. It follows that, for odd primes $t$ with $t \neq 3,5,7,31$, we must have $m=1$; that is, $W(\mathcal{D}) / N \simeq C_{2}($ and $N=\widetilde{N})$.

For $\mathcal{K}=\{3,3,4,3\}_{(t, t, 0,0)}$, we can instead use a "translation" $\gamma$ (say) of $\mathcal{K}$ which maps $i_{4}$ to $i_{5}$ or $i_{6}$. Then $r(\gamma)$ cannot be trivial if $m=5$ or 6 . Hence for odd primes $t$ with $t \neq 3,5,7,31$ we must have $m \leq 4$, and thus $\left\{\{4,3,3,4\}_{(2,2,0,0)}, \mathcal{K}\right\}$ cannot exist.

Identification vector $\left(2^{n-1}\right)$. Finally we discuss quotients whose facets are $\left\{4,3^{n-3}, 4\right\}_{\left(2^{n-1}\right)}$, with identification vector $\left(2^{n-1}\right)$. This is the hardest of the three cases. In our examples, we have $n=4$ or 5 . If $\mathcal{F}=\{3,4\}$, then in $A\left(2^{\mathcal{F}}\right)$ we have

$$
\left(\rho_{0} \rho_{1} \rho_{2} \rho_{3}\right)^{6}=\sigma_{0} \sigma_{1} \cdots \sigma_{5}=\sigma_{0} \sigma_{3} \cdot \sigma_{1} \sigma_{4} \cdot \sigma_{2} \sigma_{5}
$$

(the order of these terms is immaterial); hence, by (5), to obtain facets $\{4,3,4\}_{(2,2,2)}$ we must choose the normal subgroup $N$ so that $\sigma_{0} \cdots \sigma_{5} \in N$. A similar remark applies to facets $\left\{4,3^{n-3}, 4\right\}_{\left(2^{n-1}\right)}$, using

$$
\left(\rho_{0} \rho_{1} \cdots \rho_{n-1}\right)^{2 n-2}=\sigma_{0} \sigma_{n-1} \sigma_{1} \sigma_{n} \cdots \sigma_{n-2} \sigma_{2 n-3} .
$$

If $F$ is a facet of $\mathcal{K}$, define $\sigma_{F}$ in $W(\mathcal{D})$ by

$$
\sigma_{F}:=\prod_{i \in F} \sigma_{i}
$$

(with the $\sigma_{i}$ in any order). Then we define

$$
\left.N:=\left\langle\varphi \sigma_{F} \varphi^{-1}\right| \varphi \in W(\mathcal{D}), F \text { a facet of } \mathcal{K}\right\rangle,
$$

the normal closure of $\sigma_{\mathcal{F}}$ in $A\left(2^{\mathcal{K}, \mathcal{D}}\right)$. As before, we have $A\left(2^{\mathcal{K}, \mathcal{D}} / N\right) \simeq W(\mathcal{D}) / N \ltimes$ $A(\mathcal{K})$. By construction, it is also clear that if the universal polytope in question does exist, then it must be isomorphic to the quotient $2^{\mathcal{K}, \mathcal{D}} / N$.

We have not been able to find the structure of the group of every regular 6polytope $\left\{\{4,3,3,4\}_{(2,2,2,2)},\{3,3,4,3\}_{\left(t^{k}, 0^{4-k}\right)}\right\}$ with $t \geq 2$ and $k=1,2$. For $(t, k)=(2,1)$, the polytope is covered by the finite polytope $\left\{\{4,3,3,4\}_{(4,0,0,0)}\right.$, $\left.\{3,3,4,3\}_{(2,0,0,0)}\right\}$ of Corollary 5.6, whose group is the semi-direct product $C_{2}^{16} \ltimes$ $[3,3,4,3]_{(2,0,0,0)}$. This fact is used in our next theorem.

Theorem 7.12. The regular 6-polytope $\left\{\{4,3,3,4\}_{(2,2,2,2)},\{3,3,4,3\}_{(2,0,0,0)}\right\}$ exists and is finite. Its group is $C_{2}^{10} \ltimes[3,3,4,3]_{(2,0,0,0)}$, of order 18874368 .

Proof. We use the geometry of $\mathcal{K}=\{3,3,4,3\}_{(2,0,0,0)}$, to show that $W(\mathcal{D}) / N \cong C_{2}^{10}$. Our first observation is that the vertices of the vertex-figure $\{3,4,3\}$ of $\mathcal{K}$ coincide in antipodal pairs, so that $\mathcal{K}$ has the same vertices as those of $\left\{\{3,3,4\},\{3,4,3\}_{6}\right\}$ (compare [17]). Recall that $\{3,4,3\}_{6}$ is the regular 4-polytope obtained from $\{3,4,3\}$ by identifying antipodal points; here the number 6 indicates the extra relation $\left(\rho_{0} \rho_{1} \rho_{2} \rho_{3}\right)^{6}=\varepsilon$ for the group $[3,4,3]_{6}$ or, equivalently, the length of the Petrie polygon (see [5]). The 48 facets of $\mathcal{K}$ occur in pairs with the same vertices, so in what follows we are really referring to 24 pairs of facets. 
For simplicity, we change our convention on the vertex labels in $\mathcal{F}$, so that we now label the 16 vertices of $\mathcal{K}$ by $0, \ldots, 15$, which we express as $a b$ in base 4 (with $a, b \in\{0, \ldots, 3\})$. Edges of $\mathcal{K}$ join two vertices with different labels $a$; vertices with the same label $a$ are antipodal in some facet. If $e=\left\{b_{0}, b_{1}\right\} \subset\{0, \ldots, 3\}$ with $b_{0} \neq b_{1}$, then we write $\bar{e}=\{0, \ldots, 3\} \backslash e$ for its complement. Then ae stands for the pair $\left\{a b_{0}, a b_{1}\right\}$ of antipodal vertices, and so on. A (double) facet will have vertices $a e$ or $a \bar{e}$, where $a=0, \ldots, 3$, and $e=\{0,1\},\{0,2\}$ or $\{0,3\}$; that is, the pairs $e$ and $\bar{e}$ always go together. The 24 (double) facets then have:

$$
\begin{array}{r}
e=\{0,1\} \text { or }\{0,2\} \quad-\text { even number of } \bar{e} \text { 's; } \\
e=\{0,3\} \quad-\text { odd number of } \bar{e} \text { 's. }
\end{array}
$$

The ten generators of $W(\mathcal{D})$ correspond to the vertices

$$
00,01,03,10,11,13,20,21,23,30 .
$$

The remainder are constructed by successively listing suitable facets:

$$
\begin{aligned}
& \{00,01,10,11,20,21,30,31\} \rightarrow 31, \\
& \{00,03,10,13,20,23,31,32\} \rightarrow 32, \\
& \{01,03,11,13,21,23,31,33\} \rightarrow 33, \\
& \{01,02,10,13,20,23,30,33\} \rightarrow 02, \\
& \{00,03,11,12,20,23,30,33\} \rightarrow 12, \\
& \{00,03,10,13,21,22,30,33\} \rightarrow 2^{2} .
\end{aligned}
$$

Bear in mind here that the product of any seven generators of $W(\mathcal{D}) / N$ corresponding to vertices of a facet is the eighth. Of course, since $\mathcal{K}$ is weakly neighbourly, all generators commute. It follows that $W(\mathcal{D}) / N$ has order at most $2^{10}$, and a tedious check shows that no nine generators suffice. Finally, for the existence of the polytope we can appeal to Lemma 2.1 and Corollary 7.5 or 7.11 , using the fact that the subgroup of $W(\mathcal{D}) / N$ induced by a facet of $\mathcal{K}$ has order $2^{7}$. This completes the proof.

We now further restrict our attention to polytopes of rank 5 with facets isomorphic to toroids $\{4,3,4\}_{(2,2,2)}$. Together with Corollaries 7.4 and 7.10 , the next theorem covers all the finite universal regular 5-polytopes of type $\{4,3,4,3\}$ (or dual type $\{3,4,3,4\})$. It is one of the most interesting examples obtained by the method of this section.

Theorem 7.13. The regular 5-polytope $\left\{\{4,3,4\}_{(2,2,2)},\{3,4,3\}\right\}$ exists and is $f$ nite. Its group is $\left(C_{2}^{6} \ltimes C_{2}^{5}\right) \ltimes[3,4,3]$, of order 2359296 ; in the semi-direct product $C_{2}^{6} \ltimes C_{2}^{5}$, the factor $C_{2}^{6}$ is its own centralizer.

Proof. Now $\mathcal{K}=\{3,4,3\}$ and $\mathcal{F}=\{3,4\}$. We show that $W(\mathcal{D}) / N \cong C_{2}^{6} \ltimes C_{2}^{5}$, a group of order $2^{11}$.

First we show that $W(\mathcal{D}) / N$ is generated by 10 involutions. Let $0, \ldots, 23$ be the vertices of $\mathcal{K}$, in such a way that $l, l+5(l=1, \ldots, 4)$ are the vertices of the cubical vertex-figure of $\mathcal{K}$ at 0 (this is a change from the previous notation). Define $I:=\{1, \ldots, 9\} \backslash\{5\}$. By the definition of $N$, if $k$ is the antipodal vertex to 0 in some facet, then $\sigma_{k} N \in\left\langle\sigma_{l} N \mid l \in I \cup\{0\}\right\rangle$. Let 5 be a vertex of the vertex-figure of the opposite vertex of $\mathcal{K}$ to 0 . We claim that

$$
W(\mathcal{D}) / N=\left\langle\sigma_{0} N, \ldots, \sigma_{9} N\right\rangle
$$


Note first that, if $i, j$ are adjacent vertices of this opposite vertex-figure, then $\{i, j, p, q, r, s\}$ is a facet for some $p, q \in I$ and $r, s$ antipodal to 0 in facets of $\mathcal{K}$. It follows that $\sigma_{i} N \in\left\langle\sigma_{l} N \mid l \in I \cup\{0, j\}\right\rangle$. Now if we migrate along the edges in the opposite vertex-figure from 5 , we get all its vertices $i$, and hence the corresponding $\sigma_{i} N$. Finally, we obtain the opposite vertex of $\mathcal{K}$ to 0 from any of the facets which contain it, whose remaining vertices are already accounted for. Hence the assertion follows.

Next we prove that the commutator subgroup has order at most 2, with $\left(\sigma_{0} \sigma_{5}\right)^{2} N$ the only possible non-trivial commutator. Clearly, $\sigma_{0} N$ commutes with $\sigma_{j} N$ for $j \in I$. Now 0 and 5 are opposite vertices in the vertex-figure of one of the vertices in $I$. Hence, by the action of $A(\mathcal{K})$ on $W(\mathcal{D}) / N$, if $\sigma_{0} N$ and $\sigma_{5} N$ commute, then so do $\sigma_{i} N$ and $\sigma_{j} N$ for all $i, j \in I$, and thus $W(\mathcal{D}) / N$ is abelian. Therefore, if $W(\mathcal{D}) / N$ is not abelian, then $\left(\sigma_{0} \sigma_{5}\right)^{2} N \neq N$.

Let $i, j$ be adjacent vertices of the opposite vertex-figure, and let $p, q, r, s$ be as above. Since $\sigma_{0}$ commutes with $\sigma_{p}, \sigma_{q}, \sigma_{r}, \sigma_{s}$, it follows that $\sigma_{0} N$ commutes with $\sigma_{p} \sigma_{q} \sigma_{r} \sigma_{s} N=\sigma_{i} \sigma_{j} N$, or $\sigma_{0} \sigma_{i} \sigma_{j} N=\sigma_{i} \sigma_{j} \sigma_{0} N$. But

$$
\sigma_{0} \sigma_{i} \sigma_{j} N=\sigma_{i}\left(\sigma_{i} \sigma_{0}\right)^{2}\left(\sigma_{0} \sigma_{j}\right)^{2} \sigma_{j} \sigma_{0} N
$$

and equating this to $\sigma_{i} \sigma_{j} \sigma_{0} N$ gives $\left(\sigma_{i} \sigma_{0}\right)^{2}\left(\sigma_{0} \sigma_{j}\right)^{2} N=N$, or

$$
\left(\sigma_{0} \sigma_{i}\right)^{2} N=\left(\sigma_{0} \sigma_{j}\right)^{2} N \text {. }
$$

Starting with the vertex 5 , and iterating such relations along edges $\{i, j\}$ of the opposite vertex-figure, gives $\left(\sigma_{0} \sigma_{j}\right)^{2} N=\left(\sigma_{0} \sigma_{5}\right)^{2} N$ for each of its vertices $j$. However, 0 and 5 are themselves antipodal vertices of the vertex-figure of one of the vertices in $I$. Thus, if we choose $l$ to be antipodal to 5 in the opposite vertex-figure, then $0,5, l$ are symmetrically related, so that

$$
\left(\sigma_{0} \sigma_{5}\right)^{2} N=\left(\sigma_{0} \sigma_{l}\right)^{2} N=\left(\sigma_{5} \sigma_{l}\right)^{2} N=\left(\sigma_{5} \sigma_{0}\right)^{2} N,
$$

or

$$
\left(\sigma_{0} \sigma_{5}\right)^{4} N=N
$$

Chasing arguments of this kind (using symmetry and connectivity properties of $\mathcal{K})$ show that $\left(\sigma_{0} \sigma_{5}\right)^{2} N$ can indeed be the only non-trivial commutator between pairs of generators $\sigma_{j} N$; it then follows that the commutator subgroup is of order at most 2. In particular, $\kappa:=\left(\sigma_{0} \sigma_{5}\right)^{2} N$ lies in the centre of $W(\mathcal{D}) / N$. Note that the 10 generators thus commute in pairs, except for the five disjoint pairs $j, j+5$ $(j=0, \ldots, 4)$, for which $\left(\sigma_{j} \sigma_{j+5}\right)^{2} N=\kappa$.

Next we prove that $W(\mathcal{D}) / N$ is not abelian, and that $W(\mathcal{D}) / N \simeq C_{2}^{6} \ltimes C_{2}^{5}$. First note that the vertex 5 in $\mathcal{K}$ is joined by an edge to exactly one vertex, 6 (say), of the vertex-figure at 0 . Let $7,8,9$ be the neighbouring vertices to 6 of this vertex-figure. If $j \in\{6, \ldots, 9\}$, then the vertices $j$ and 5 lie in a common facet, so that $\sigma_{j} N$ and $\sigma_{5} N$ commute. It follows that $B:=\left\langle\sigma_{5} N, \ldots, \sigma_{9} N\right\rangle$ is an abelian subgroup generated by (at most) 5 involutions.

Let $Z:=W(\mathcal{D}) / N$ and $\alpha_{j}:=\sigma_{j} N$ for $j=0, \ldots, 9$. Then for all $i, j$, either $\alpha_{j} \alpha_{i} \alpha_{j}=\alpha_{i}$ or $\alpha_{j} \alpha_{i} \alpha_{j}=\kappa \alpha_{i}$. Now $A:=\left\langle\alpha_{0}, \ldots, \alpha_{4}, \kappa\right\rangle$ is a normal abelian subgroup of $Z$ generated by (at most) 6 involutions, and the canonical projection $\pi: Z \rightarrow Z / A$ takes $B:=\left\langle\alpha_{5}, \ldots, \alpha_{9}\right\rangle$ onto $Z / A$. In particular, $|Z| \leq 2^{11}$.

Assume for the moment that we indeed have $|Z|=2^{11}$. Then $A \simeq C_{2}^{6}$ and $B \simeq Z / A \simeq C_{2}^{5}$, because the numbers of generators of $A$ and $B$ are at most 6 and 
5 , respectively. It follows that $Z=A \ltimes B \simeq C_{2}^{6} \ltimes C_{2}^{5}$. Consider now how $B$ acts on $A$ by conjugation. Here it is helpful to observe that we can identify $A$ with a 6 -dimensional vector space over $G F(2)$, with basis vectors $\alpha_{j}$ for $j=0, \ldots, 4$ and $\kappa$ identified with those of the standard column basis, and represent conjugation with an element in $B$ by a $6 \times 6$ matrix over $G F(2)$. Then it is immediate that the generators $\alpha_{j}(j=5, \ldots, 9)$ correspond to the matrices

$$
\left[\begin{array}{cc}
I_{5} & 0 \\
e_{j-5} & 1
\end{array}\right],
$$

with $I_{5}$ the $5 \times 5$ identity matrix, and $e_{0}, \ldots, e_{4}$ the standard row basis of $G F(2)^{5}$. In particular, this implies that conjugation gives a faithful representation $B \rightarrow$ $\operatorname{Aut}(A)$, or, equivalently, that $A$ is its own centralizer in $Z$.

On the other hand, we can now complete the proof by observing that there is indeed a specific group $C_{2}^{6} \ltimes C_{2}^{5}$, with the action of $C_{2}^{5}$ on $C_{2}^{6}$ defined by (42), which satisfies the given relations. Hence there can be no possibility of our group $Z$ collapsing onto a group of order smaller than $2^{11}$, since $Z$ is just determined by these relations. The existence of the polytope now follows from Lemma 2.1 and Corollary 7.4 or 7.10 , using the fact that the subgroup of $W(\mathcal{D}) / N$ induced by a facet of $\mathcal{K}$ has order $2^{5}$.

We conclude the paper with an application of our method to the projective 4polytope $\{3,4,3\}_{6}$, giving us 5 -polytopes with toroidal facets and projective vertexfigures (for the notation, see above).

Theorem 7.14. For $k=1,2,3$, the 5-polytope $\left\{\{4,3,4\}_{\left(2^{k}, 0^{3-k}\right)},\{3,4,3\}_{6}\right\}$ exists and is finite. Its group is $C_{2}^{m(k)} \ltimes[3,4,3]_{6}$, with $m(k)=3,5,8$ as $k=1,2,3$, respectively.

Proof. Let $\mathcal{K}:=\{3,4,3\}_{6}$. Then Theorem 7.1 gives the polytope for $k=1$, with group $C_{2}^{3} \ltimes A(\mathcal{K})$; here we use the fact that $\omega\left(\mathcal{G}_{\mathcal{K}}\right)=3$. If $k=2$, Theorem 7.8 suggests that the group should be $C_{2}^{5} \ltimes A(\mathcal{K})$. Indeed, as in the proof of Corollary 7.9, we can use the representation $A(\mathcal{K}) \mapsto G L_{5}(2)$ defined by (40) (with $n=4$ ) to identify $C_{2}^{5} \ltimes A(\mathcal{K})$ as the group of the regular polytope $\left\{\{4,3,4\}_{(2,2,0)}, \mathcal{K}\right\}$.

Last, let $k=3$. Since $\mathcal{K}$ is weakly neighbourly, $W(\mathcal{D}) / N$ is an elementary abelian 2 -group. Let $1, \ldots, 8$ be the vertices of the vertex-figure at 0 , with opposite faces $\{1, \ldots, 4\},\{5, \ldots, 8\}$ (again, we have changed our earlier notation). Let 9 be such that $\{0, \ldots, 4,9\}$ is a facet of $\mathcal{K}$. Then $\{0,5, \ldots, 9\}$ is also a facet of $\mathcal{K}$. Thus we can choose $\sigma_{0} N, \ldots, \sigma_{7} N$ as generators, obtaining $\sigma_{9} N$ from the first facet and $\sigma_{8} N$ from the second. We obtain the two remaining generators $\sigma_{10} N$ and $\sigma_{11} N$ as we found $\sigma_{9} N$. Clearly, fewer generators will not serve, so that $W(\mathcal{D}) / N \simeq C_{2}^{8}$. Finally, since the subgroup of $W(\mathcal{D}) / N$ induced by a facet of $\mathcal{K}$ has order $2^{5}$, Lemma 2.1 now proves the existence of the polytope.

For most of the polytopes constructed in this section, the group order has been checked by Asia Weiss using the Coxeter-Todd algorithm. The authors are indebted to her for this assistance. 
2. H.S.M. Coxeter, Regular skew polyhedra in 3 and 4 dimensions and their topological analogues. Proc. London Math. Soc. (2) 43 (1937), 33-62. Reprinted with corrections in Twelve Geometric Essays, Southern Illinois University Press (Carbondale, 1968), 75-105. MR 46:9843

3. H.S.M. Coxeter, Groups generated by unitary reflections of period two. Canadian J. Math. $\mathbf{9}$ (1957), 243-272. MR 19:248

4. H.S.M. Coxeter, Regular honeycombs in hyperbolic space. Proc. Internat. Congr. Math. (Amsterdam, 1954), Vol. III, Noordhoff, Groningen, and North-Holland, Amsterdam (1956), 155169. Reprinted with corrections in Twelve Geometric Essays, Southern Illinois University Press (Carbondale, 1968), 199-214. MR 19:304 MR 46:9843

5. H.S.M. Coxeter, Regular Polytopes (3rd edition), Dover (New York, 1973). MR 51:6554

6. H.S.M. Coxeter and W.O.J. Moser, Generators and Relations for Discrete Groups (4th edition) Springer (Berlin, 1980). MR 81a:20001

7. H.S.M. Coxeter and G.C. Shephard, Regular 3-complexes with toroidal cells. J.Combinatorial Theory B 22 (1977), 131-138. MR 55:11140

8. L. Danzer and E. Schulte, Reguläre Inzidenzkomplexe I. Geom. Ded. 13 (1982), 295-308. MR 84h:51042

9. A.W.M. Dress, Regular polytopes and equivariant tessellations from a combinatorial point of view. In Algebraic Topology (Göttingen 1984), Lecture Notes in Mathematics 1172, Springer (1985), 56-72. MR 87i:52025

10. B. Grünbaum, Regularity of graphs, complexes and designs. In Problèmes combinatoire et théorie des graphes, Coll. Int. CNRS No.260 (Orsay, 1977), 191-197. MR 81f:05060

11. A.I. Malcev, On faithful representations of infinite groups of matrices. Mat. Sb. 8 (1940), 405-422 (Russian). English translation in Amer. Math. Soc. Transl. Ser. (2) 45 (1965), 1-18. MR 22:216

12. P. McMullen, Combinatorially regular polytopes. Mathematika 14 (1967), 142-150. MR 36: 4436

13. P. McMullen, Realizations of regular polytopes. Aequationes Math. 37 (1989), 38-56. MR 90c:52014

14. P. McMullen, Locally projective regular polytopes. J. Combinatorial Theory A 65 (1994), 1-10. MR 95a:52015

15. P. McMullen and E. Schulte, Constructions of regular polytopes. J. Combinatorial Theory A 53 (1990), 1-28. MR 91c:52017

16. P. McMullen and E. Schulte, Regular polytopes from twisted Coxeter groups. Math. Zeitschrift 201 (1989), 209-226. MR 90g:51031

17. P. McMullen and E. Schulte, Regular polytopes from twisted Coxeter groups and unitary reflexion groups. Advances Math. 82 (1990), 35-87. MR 92f:52022

18. P. McMullen and E. Schulte, Hermitian forms and locally toroidal regular polytopes. Advances Math. 82 (1990), 88-125. MR 91j:52013

19. P. McMullen and E. Schulte, Regular polytopes of type $\{4,4,3\}$ and $\{4,4,4\}$. Combinatorica 12 (1992), 203-220. MR 93h:52014

20. P. McMullen and E. Schulte, Finite quotients of infinite universal polytopes. In Discrete and Computational Geometry (ed. J. Goodman, R. Pollack and W. Steiger), DIMACS Series, Vol. 6 (AMS-ACM, 1991), 231-236. MR 92j:52017

21. P. McMullen and E. Schulte, Higher toroidal regular polytopes. Advances Math. (to appear).

22. P. McMullen and E. Schulte, Quotients of polytopes and C-groups. Discrete Comput. Geom. 11 (1994), 453-464. MR 95h:52010

23. P. McMullen and E. Schulte, Abstract Regular Polytopes (monograph in preparation).

24. B. Monson and A.I. Weiss, Regular 4-polytopes related to general orthogonal groups. Mathematika 37 (1990), 106-118. MR 91k:52021

25. E. Schulte, Reguläre Inzidenzkomplexe II. Geom. Ded. 14 (1983), 33-56. MR 85d:51006

26. E. Schulte, Amalgamations of regular incidence-polytopes. Proc. London Math. Soc. (3) $\mathbf{5 6}$ (1988), 303-328. MR 88k:51044

27. E. Schulte, On a class of abstract polytopes constructed from binary codes. Discrete Math 84 (1990), 295-301. MR 91j:52014

28. E. Schulte, Classification of locally toroidal regular polytopes. In Polytopes: Abstract, convex and computational (ed. T. Bisztriczky et al.), NATO ASI Series C 440, Kluwer (Dordrecht, 1994), 125-154. MR 95i:52001 
29. J. Tits, Buildings of spherical type and finite BN-pairs. Lecture Notes in Mathematics $\mathbf{3 8 6}$, Springer (Berlin, 1974). MR 57:9866

30. A.I. Weiss, An infinite graph of girth 12. Trans. Amer. Math. Soc. 283 (1984), 575-588. MR 85j:52023

31. A.I. Weiss, Incidence-polytopes of type $\{6,3,3\}$. Geom. Ded. 20 (1986), 147-155. MR 87e:52013

Department of Mathematics, University College London, Gower Street, London WCIE 6BT, ENGLAND

E-mail address: p.mcmullen@@ucl.ac.uk

Department of Mathematics, Northeastern University, Boston, Massachusetts 02115

E-mail address: schulte@@neu.edu 